OPEN ACCESS

Edited by:

Peter Igaz,

Semmelweis University, Hungary

Reviewed by:

Poonam Ramsevak Pandey, National Institutes of Health (NIH),

United States

Peter Szabo,

Bristol Myers Squibb (United States),

United States

*Correspondence:

Yongguang Gao

gaoyongguang@nwpu.edu.cn

Airong Qian

qianair@nwpu.edu.cn

tThese authors have contributed equally to this work

Specialty section:

This article was submitted to

RNA

a section of the journal

Frontiers in Genetics

Received: 16 July 2020

Accepted: 30 September 2020

Published: 13 November 2020

Citation:

Patil S, Dang K, Zhao X, Gao Y and Qian A (2020) Role of LncRNAs and CircRNAs in Bone Metabolism

and Osteoporosis.

Front. Genet. 11:584118.

doi: $10.3389 /$ fgene.2020.584118

\section{Role of LncRNAs and CircRNAs in Bone Metabolism and Osteoporosis}

\author{
Suryaji Patil1', Kai Dang ${ }^{1+}$, Xin Zhao ${ }^{2}$, Yongguang Gao ${ }^{1,3 *}$ and Airong Qian ${ }^{1 *}$ \\ ${ }^{1}$ Lab for Bone Metabolism, Xi'an Key Laboratory of Special Medicine and Health Engineering, Key Lab for Space \\ Biosciences and Biotechnology, Research Center for Special Medicine and Health Systems Engineering, NPU-UAB Joint \\ Laboratory for Bone Metabolism, School of Life Sciences, Northwestern Polytechnical University, Xi'an, China, ${ }^{2}$ School \\ of Pharmacy, Shaanxi Institute of International Trade \& Commerce, Xi'an, China, ${ }^{3}$ Department of Chemistry, Tangshan \\ Normal University, Tangshan, China
}

Bone is a mechanosensitive organ that provides strength and support. Many bone cells, various pathways, and signaling molecules coordinate bone metabolism and also determine the course of bone diseases, such as osteoporosis, osteonecrosis, osteopenia, etc. Osteoporosis is caused by increased bone resorption and reduced bone formation due to the changes in the level of different proteins and RNAs in osteoclast or/and osteoblasts. The available therapeutic interventions can significantly reduce bone resorption or enhance bone formation, but their prolonged use has deleterious side effects. Therefore, the use of non-coding RNAs as therapeutics has emerged as an interesting field of research. Despite advancements in the molecular field, not much is known about the role of long non-coding RNAs (IncRNAs) and circular RNAs (circRNAs) in bone homeostasis and osteoporosis. Therefore, in this article, we summarize the role of IncRNAs and circRNAs in different bone cells and osteoporosis so that it might help in the development of osteoporotic therapeutics.

Keywords: bone metabolism, long ncRNAs (IncRNAs), circular RNA (circRNAs), osteoporosis, therapeutics

\section{INTRODUCTION}

Osteoporosis is characterized by reduced bone mass, weakened microarchitecture, and risk of fractures, affecting both women and men (Lane et al., 2000). More than 200 million people are estimated to be suffering from osteoporosis worldwide. In their lifetime, 1 in 3 women and 1 in 5 men worldwide become susceptible to osteoporotic fractures, making it one of the lifethreatening diseases (Sözen et al., 2017). Different factors contribute to the pathophysiology of osteoporosis, such as defects in trabecular microarchitecture, inability to repair damage caused by normal activities, and increased rate of bone remodeling (Armas and Recker, 2012). Dualenergy X-ray absorptiometry (DEXA) and quantitative computed tomography (CT) scans are the common diagnostic techniques used to estimate bone mass and risk of fractures (Lane et al., 2000). Pharmaceutical treatments available for osteoporosis include calcium and vitamin $\mathrm{D}$, calcitonin, estrogen replacement therapy (ERT), etidronate, fluoride, intermittent parathyroid hormone therapy (iPHT), and bisphosphonates alendronate (ALN) and risedronate (RIS) to reduce the bone resorption or increase bone formation (Prestwood et al., 1995; Eiken and Vestergaard, 2016). Despite these advantages, these pharmaceutical agents alone or in combination have limitations, such as low efficacy in reducing non-vertebral fracture and adverse effects of long-term application (Tabatabaei-Malazy et al., 2017). Therefore, the identification and development of new therapeutics based on gene therapy, which employs the use of various methods to deliver different nucleic 


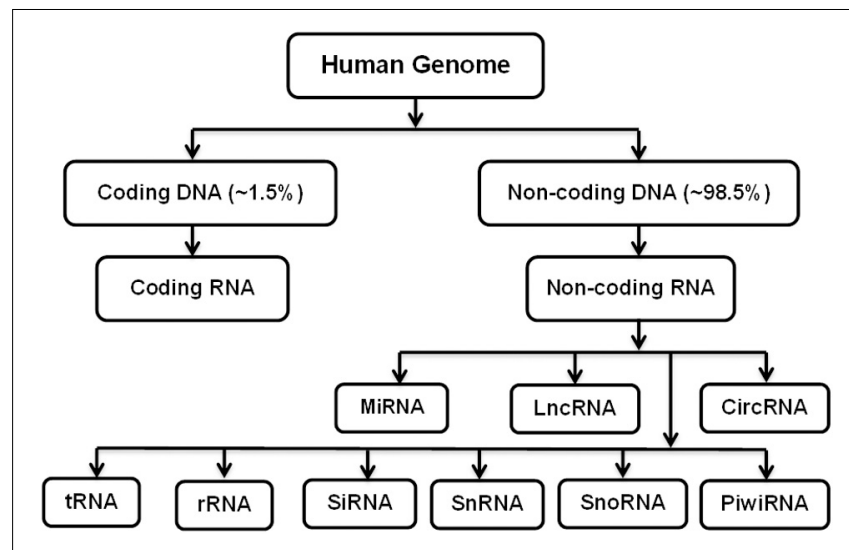

FIGURE 1 | Coding and Non-coding RNAs. the target gene is regulated by lncRNAs through cis- or transregulation (Chi et al., 2019). Due to their ability to epigenetically control the expression of various genes in physiological as well as pathological processes makes them a critical factor in human diseases, such as osteoporosis (Wu et al., 2018b; Chi et al., 2019; Yu et al., 2019).

\section{LncRNAs in Bone Mesenchymal Stem Cell Regulation}

Mesenchymal stem cells (MSCs) provide a vital source in regenerative medicine as well as cell therapies in advancedage patients. Bone marrow MSCs (BMSCs) provide support for hematopoietic cells as well as play an important part in bone formation, and this distinctive ability makes them unique and possibly sensitive to age-related diseases (Baker et al., 2015). Therefore, it is important to study the molecular mechanism involved in BMSC regulation (Table 1).

In cancer cells, IncRNA HOX transcript antisense RNA (HOTAIR) is known to regulate gene expression by mediating the assembly of chromatin modifiers (Loewen et al., 2014). In MSC regulation, upregulated HOTAIR is shown to inhibit MSC differentiation into adipogenic cells by modulating RNA-DNADNA triple helix formation, and increasing cell passage could hypermethylate the HOTAIR binding sites at genomic regions, facilitating this triple helix formation (Kalwa et al., 2016). Thus, through epigenetic modifications, HOTAIR could regulate the MSC differentiation Antisense lncRNAs are also involved in regulating osteogenic differentiation of human mesenchymal stem cells (hMSC)s, such as semaphorin 3B-Antisense 1 (SEMA3B-AS1). The lentivirus-mediated upregulation of SEMA3B-AS1 is revealed to downregulate the expression of proteins involved in the actin cytoskeleton and focal adhesion (FA) as well as those that are involved in extracellular matrixreceptor interaction. This downregulation inhibited proliferation and osteogenic differentiation of hMSCs (Zhang et al., 2019a). Another antisense lncRNA involved in bone metabolism is lncRNA brain-derived neurotrophic factor antisense (BDNFAS). During the osteogenic differentiation of BMSCs, the expression of IncRNA BDNF-AS was elevated. A gain-offunction experiment shows BDNF-AS prompted BMSCs to proliferate but did not promote osteogenic differentiation. Furthermore, it is suggested that the upregulation of BDNF-AS downregulated the levels of BDNF, osteopontin (OPN), and runt-related transcription factor 2 (Runx2), thus reducing osteogenic differentiation (Feng et al., 2018b).

The detection of initial BMSC differentiation into osteogenic cells is generally measured by the activity of alkaline phosphatase (ALP) in vitro and also serves as an important factor in the calcification of BMSCs (Chen et al., 2019c). It has been described that the high expression of lncRNA X-inactive specific transcript (XIST) had a negative effect on ALP and Runx2 expression and osteoblast differentiation in BMSCs (Chen et al., 2019c). The high expression of lncRNA AK016739 in BMSCs has also been described to negatively correlate with marker genes of osteogenic differentiation. This high expression of AK016739 inhibited osteoblast differentiation, whereas in vivo 
TABLE 1 | LnCRNAs involved in BMSC regulation.

\begin{tabular}{|c|c|c|c|c|}
\hline LncRNA & Relative expression & Role & Target & References \\
\hline HOTAIR & Upregulation & Inhibits adipogenic differentiation of MSCs & DNA methylation & Kalwa et al., 2016 \\
\hline SEMA 3B-AS1 & Upregulation & Inhibits proliferation and osteogenic differentiation of hMSCs & Proteins in actin cytoskeleton, FA & Zhang et al., 2019a \\
\hline XIST & Upregulation & Inhibits osteoblast differentiation & Runx2, ALP & Chen et al., 2019c \\
\hline AK016739 & Downregulation & Promotes bone formation in osteoporosis mice & - & Yin et al., 2019 \\
\hline BDNF-AS & Upregulation & Inhibits osteogenic differentiation of BMMSCs & BDNF & Feng et al., 2018b \\
\hline Bmncr & Upregulation & Stimulates bone formation in vivo & fibromodulin & Li et al., 2018a \\
\hline Bmcob & Upregulation & Promotes osteoblastic differentiation of BMSC & SBP2 & Sun et al., 2018 \\
\hline NEF & Upregulation & Promotes osteoporosis & IL-6 & Ma et al., 2019 \\
\hline
\end{tabular}

"-" Unknown.

inhibition rescued the bone formation in ovariectomized (OVX) osteoporosis mice (Yin et al., 2019). According to Li et al., lncRNA, bone marrow associated ncRNA (BMNCR) could also regulate the fate of BMSCs through fibromodulin, an extracellular matrix protein, and activating the bone morphogenetic protein (BMP) pathway (Li et al., 2018a). The deficiency of BMNCR has been reported to decrease bone mass, whereas the upregulation could stimulate bone formation and lower adipogenesis in the bone marrow. Moreover, BMNCR provided the scaffold to enhance the assembly of a transcriptional coactivator with a PDZ-binding motif (TAZ) and ABL to form TAZ and Runx2/peroxisome proliferator-activated receptor gamma (PPARG) transcriptional complexes, which are required for bone formation ( $\mathrm{Li}$ et al., 2018a). Many enzymes protect the bone from oxidative stress, such as glutathione peroxidases (GPxs) and thioredoxin reductases (TrxRs), which play a crucial role in regulating bone homeostasis as well as reducing bone loss (Zhang et al., 2014). These enzymes contain selenocysteine, and selenocysteine insertion sequence (SECIS) binding protein 2 (SBP2) is an essential transacting factor required for the cotranslational insertion of selenocysteine into selenoproteins (Dumitrescu et al., 2010). It is reported that LncRNA Bmcob could control the transport of SBP2 between the nucleus and cytoplasm, and when overexpressed, Bmcob could regulate the expression of selenoproteins, sepp1, and increase osteogenesis of BMSCs (Sun et al., 2018). LncRNA-Neighboring enhancer of FOXA2 (NEF), which is known to the inhibit Wnt/ $\beta$-catenin signaling and transforming growth factor- $B$ TGF- $\beta$ pathways in cancer cells (Liang et al., 2018; Ju et al., 2019) is also reported in postmenopausal osteoporosis (PMOP) patients in a high concentration. In human BMSCs isolated from PMOP, enforced expression of NEF inhibited the secretion of IL-6, indicating its potential not only in diagnostics, but also as a prognostic biomarker for PMOP (Ma et al., 2019).

\section{LncRNA Involved in BMSC Regulation Through miRNA}

Due to their intrinsic ability of self-renewal and differentiation, BMSCs can be an ideal choice in treating osteoporosis. The available pharmaceutical interventions, though they have efficacy, also have side effects. Glucocorticoids are considered as standard therapy not only for reducing inflammation, but also for activation of the immune system in various diseases. Nevertheless, glucocorticoids have severe side effects on many organ systems, such as gastrointestinal, cardiovascular, endocrine, musculoskeletal, etc. (Oray et al., 2016). The most common cause of glucocorticoid administration is secondary osteoporosis and non-traumatic osteonecrosis (Weinstein, 2012). Glucocorticoid-treated BMSCs, when induced for differentiation, have shown low levels of TCONS 00041960 lncRNA. When overexpressed, TCONS 00041960 had a positive effect on the expression of osteogenic genes but not on the expression of adipocyte-specific genes. Moreover, TCONS 00041960 interactions with miR-204-5p and miR-125a-3p regulated not only Runx2, but also glucocorticoid-induced leucine zipper (GILZ), an antiadipogenic gene to control the differentiation fate of BMSCs treated with glucocorticoid (Shang et al., 2018). During osteogenic differentiation of BMSCs, lncRNA MSC antisense RNA 1 (MSC-AS1) and BMP2 were significantly upregulated. The $3^{\prime}$ UTR of MSC-AS1 and BMP2 genes have been reported to act as a docking site for miR-140-5p. The inhibition of MSC-AS1 could decrease the expression of genes associated with osteogenesis as well as miR-140-5p, whose knockdown reduced BMP2 expression, but inhibition of both MSC-AS1 and miR-140-5p could rescue this inhibitory effect. Thus, miR-140-5p could not bind to BMP2, and MSC-AS1 could stimulate the osteogenic differentiation of BMSCs to ease osteoporosis progression (Zhang et al., 2019c). Similarly, LncRNA KCNQ1OT1 expression was also increased during osteogenic differentiation. It is shown that, when KCNQ1OT1 was upregulated, it targeted miR-214, which is known to bind to 3'UTR of BMP2. Therefore, by targeting miR-214, KCNQ1OT1 positively regulated the osteogenic differentiation of BMSCs by upregulating BMP2 expression (Wang et al., 2019a; Figure 2).

The telomere is essential for cell survival, and it is maintained by the enzyme telomerase that functions by adding guaninerich repetitive sequences to maintain the length of telomeres (Zvereva et al., 2010). To maintain the activity of telomerase, the template sequence is offered by LncRNA TERC (telomerase RNA elements). According to Gao et al. (2020a), upregulation of lowered TERC could target miR-217, lower its levels, and indirectly increase Runx2, thus promoting osteogenesis and reducing the progression of osteoporosis. According to another study, an lncRNA, growth arrest-specific 5 (GAS5), was also present at a low concentration in osteoporosis patients and could also upregulate Runx2. When overexpressed, GAS5 increased hMSC osteogenic differentiation by targeting miR-498, which is 


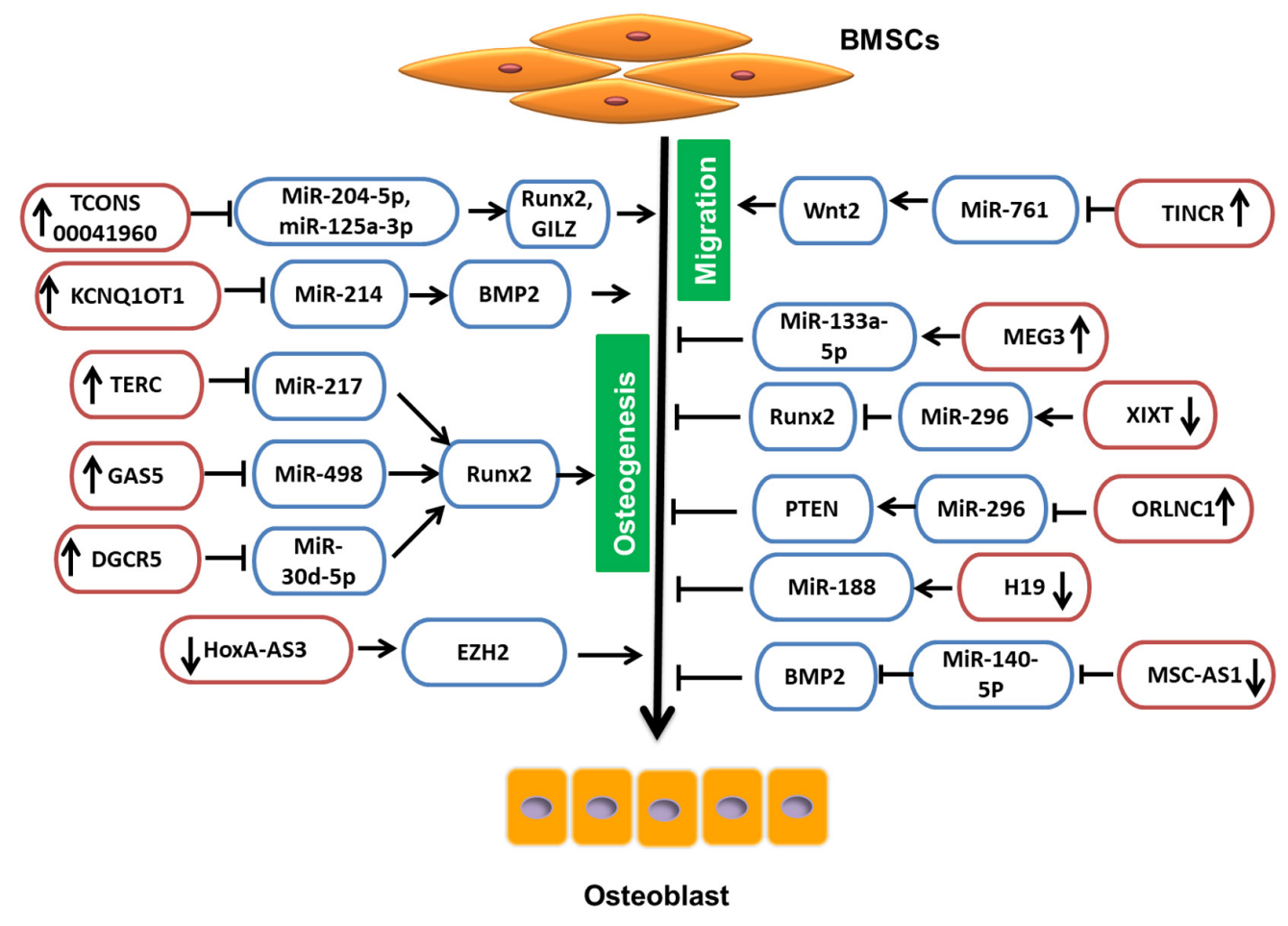

FIGURE 2 | LncRNA involved in BMSC regulation by targeting miRNAs.

known to target Runx 2 and lower osteogenesis. Thus, by targeting miR-498, GAS5 could promote bone formation (Feng et al., 2019). Similarly, the study by $\mathrm{Wu}$ et al. reports the upregulation of lncRNA DiGeorge syndrome critical region gene 5 (DGCR5) in hMSCs derived from PMOP patients could promote hMSCs to differentiate to osteoblasts, increasing Runx2 expression though miR-30d-5p (Wu et al., 2018c).

LncRNA small nucleolar RNA host gene 1 (SNHG1) sponges the action of the number of miRNAs in cancer cells; similarly, lncRNA also plays an important role in the differentiation of osteoblasts and osteoclasts. The change in these miRNAs could lead to the loss of ability, which may indicate the possible involvement of SNHG1 in osteoporosis (Huang et al., 2019a). The overexpression of lncRNA SNHG1 is shown to reduce the differentiation ability of BMSCs by promoting the interaction between neural precursor cell expressed developmentally downregulated protein 4 (Nedd4) and p-p38 and p-p38 ubiquitination (Jiang et al., 2019b). Nedd4is an E3-ubiquitin ligase involved in the ubiquitination of membrane proteins and in regulating the availability of ion channels and transporter membrane proteins during signaling as well as enhancing bone formation (Jeon et al., 2018; Manning and Kumar, 2018). Thus, silencing of SNHG1 increased the bone mineral density (BMD) and levels of osterix (OSX) protein in OVX mice (Jiang et al., 2019b).

In the pathogenesis and development of PMOP, lncRNAs also play a significant role. The study reports that the enforced lncRNA maternally expressed gene 3 (MEG3) expression is positively correlated with the expression of miR-133a-3p in
BMSCs derived from PMOP. This regulated expression of miR-133a-5p by MEG3 inhibited BMSC differentiation into osteoblasts (Wang et al., 2017a). Also, according to Zhuang et al. (2015), in MSCs isolated from multiple myeloma (MM), lncRNA MEG3 was present in a relatively low concentration during osteogenic differentiation. When MEG3 was knocked down, considerably lower expression of osteogenic markers as well as BMP4 were observed, whereas upregulation increased their expression (Zhuang et al., 2015). Another study on MSCs revealed that the IncRNA HOXA cluster antisense RNA 3 (HoxAAS3) level was proportional to the adipogenic induction of MSC. However, the silencing of HoxA-AS3 in MSCs increased osteogenesis. Furthermore, the study revealed that HoxA-AS3 could interact with the enhancer of zeste $2(\mathrm{EZH} 2)$ that is essential for Runx2 H3 lysine-27 trimethylation (H3K27me3). Thus, through epigenetic control, HoxA-AS3 could determine the fate of MSCs (Zhu et al., 2016). Metastasis-associated lung adenocarcinoma transcript 1 (MALAT1) is one of the lncRNAs that is associated with human diseases and is a major player in the development and pathogenesis of various cancers. In various mammalian species, MALAT1 is evolutionarily conserved and highly expressed (Zhang et al., 2017). LncRNA MALAT1 has also been reported in osteoporosis. MiR-143, which targeted the $3^{\prime}$-UTR of OSX, is reported to be the target of MALAT1. In addition, inhibition of MALAT1 is shown to downregulate the expression of OSX and reduces osteogenic differentiation, and overexpression could increase osteogenic differentiation (Gao et al., 2018), whereas, according to Zhang et al., inhibition of lncRNA MALAT1 is shown to reduce ALP activity in 
BMSCs and inhibit osteogenic differentiation of BMSCs via the MAPK signaling pathway (Zheng et al., 2019). Adipose-derived mesenchymal stem cells (ADSCs) act as a vital source of stem cells in tissue repair and regeneration and are shown to be regulated by MALAT1. The expression of MALAT1 has been reported to promote osteogenic differentiation of ADSCs by increasing Runx2 expression. Furthermore, a bifluorescein assay reveals that MALAT1 could target miR-30, which inhibits Runx2 (Yi et al., 2019).

For many medical conditions as well as fractures, glucocorticoids, such as dexamethasone (Dex), are commonly prescribed, but their long-term use leads to glucocorticoidinduced osteoporosis (GIOP; Whittier and Saag, 2016; Adami and Saag, 2019). One study has described that, when osteoblastic cell lines (OB-6 and hFOB1.19) were treated with Dex, the expression of IncRNA MALAT1 was downregulated. Furthermore, it showed that lentivirus-mediated upregulation of MALAT1 promoted cell viability and survival and inhibited PPM1E (protein phosphatase, $\mathrm{Mg}^{2+} / \mathrm{Mn}^{2+}$-dependent $1 \mathrm{E}$ ), which activated AMPK signaling (Fan et al., 2018). Moreover, overexpression of MALAT1 also stimulated nicotinamide adenine dinucleotide phosphate (NADPH) activity and activation of $\mathrm{Nrf2}$ signaling, whereas silencing intensified cytotoxicity induced by Dex. Thus, MALAT1 could shield the osteoblasts from Dex injury to promote cell survival (Fan et al., 2018). LncRNA epigenetically induced lncRNA 1 (EPIC1) has also been described by Zhang et al. (2018) to provide a protective effect on the survival of Dex-treated osteoblasts. The work has shown that the upregulation of EPIC1 stimulated cell survival and targeted Myc while silencing aggravated cytotoxicity induced by Dex (Zhang et al., 2018). Thus, through targeting different molecules and pathways, IncRNAs could regulate proliferation, differentiation, and functions of osteoblasts and even provide protection.

The osteoporosis-related lncRNA 1 (ORLNC1), which was highly expressed not only in BMSCs or the serum of osteoporotic mice, but also in bone tissue of osteoporotic patients, could decrease the osteogenic differentiation of BMSCs (Yang et al., 2019). Moreover, ORLNC1 is demonstrated to be a competitive endogenous RNA (ceRNA) for miR-296, which, by targeting phosphatase and tensin homolog (PTEN), a well-known negative regulator of osteogenesis could reduce osteogenesis (Yang et al., 2019).

Bone morphogenetic protein 1 (BMP1) is a metalloprotease that plays an important role in various cellular functions and acts as an extracellular matrix protein. It is vital in the process of osteogenesis, and its deficiency can increase bone fragility (Asharani et al., 2012). BMSCs isolated from osteoporosis patients show low expression of BMP1, which is regulated in miR-29b-3p. LncRNA nuclear enriched abundant transcript 1 (NEAT1) is shown to target miR-29b-3p and increase BMP1 in hBMSCs to promote osteogenic differentiation (Zhang et al., 2019e). Conversely, lncRNA XIXT is reported to reduce osteogenic differentiation of hBMSCs in vitro by reducing Runx 2 through miR-30a-5p (Zhang et al., 2019b).

At the start of bone repair, an inflammatory response plays an important role in employing MSCs and directing not only their migration, but also differentiation, to speed up the process of bone repair (Liu et al., 2018). Therefore, it is important to understand the molecular mechanisms involved in regulating cell migration. Terminal differentiation-induced lncRNA (TINCR) has been reported in the proliferation and apoptosis as well as in cell migration (Zheng et al., 2020). Moreover, TINCR upregulation promoted cell migration of rat MSCs and also reduced miR-761, which targeted Wnt2. Thus, TINCR acted as a competitive endogenous RNA to increase the Wnt2 to promote migration of rat MSCs (Zheng et al., 2020). According to the study, MCF2L-AS1 was highly expressed during BMSC osteogenic differentiation, and knockdown could enhance the expression of miR-33a and reduce Runx 2 expression. This could lead to inhibition of osteoblast differentiation. Thus, MCF2LAS1 stimulated osteogenic differentiation in BMSCs by positively regulating the expression of Runx2 (Chen et al., 2020).

Long non-coding RNA, H19 was originally defined as an oncofetal transcript, expressed from the maternal allele (Ghafouri-Fard et al., 2020). In malignant tumors, it is well known that H19 is aberrantly expressed and regulates cell proliferation, migration, invasions, antiapoptosis, etc., as well as acts as a microRNA sponge to indirectly regulate the expression of microRNA target genes (Ye et al., 2019). Wang et al. (2018a) have described that the lncRNA H19 and Liganddependent co-repressor (LCoR) were upregulated in mBMSCs under osteogenic induction, and miR-188 was downregulated, which regulated LCoR in mBMSCs. Subsequent investigation has elucidated that, when $\mathrm{H} 19$ was silenced, the levels of miR188 were increased, leading to reduced osteogenic differentiation in mBMSCs (Wang et al., 2018a). LncRNA H19 has also been reported to be influenced by mechanical tension. The mechanical strain could promote BMSCs for osteogenic differentiation as well as the expression of lncRNA H19 and lower the expression of miR-138. The deficiency of H19, however, reduced this mechanical tension-induced differentiation of BM-MSCs to osteoblasts and enhanced miR-138 expression, which weakened the focal adhesion kinase (FAK; Wu et al., 2018a). According to Huang et al. (2015), upregulation of $\mathrm{H} 19$ promoted in vitro osteogenic differentiation of hMSCs and bone formation in vivo. Furthermore, H19 along with miR-675 reduced the expression of transforming growth factor- $\beta 1$ (TGF- $\beta 1$ ), which inhibited Smad3 phosphorylation as well as histone deacetylase (HDAC) $4 / 5$ to enhance expression of the osteoblast gene (Huang et al., 2015). Thus, by targeting different microRNAs, lncRNAs could regulate the different aspects of BMSCs and, consequently, affect osteogenesis (Table 2).

\section{LncRNA Involved in BMSC Regulation Through Wnt $/ \beta$-Catenin Signaling}

$\mathrm{Wnt} / \beta$-Catenin signaling is an important pathway that influences the function of different cells, which is governed and regulated by different factors, such as proteins, miRNAs, and lncRNAs. Various $\operatorname{lncRNAs}$ regulate the $\mathrm{Wnt} / \beta$-Catenin signaling, such as LncRNA H19, LncRNA LINC00707, LncRNA linc-ROR, LncRNA AK045490, etc., through different mechanisms and targets (Figure 3).

Long non-coding RNA H19 upregulation is reported to function as a competing endogenous RNA for miR-141 and 
TABLE 2 | LncRNA involved in BMSC regulation by targeting miRNAs.

\begin{tabular}{|c|c|c|c|c|}
\hline LncRNA & Relative expression & Role & Target & References \\
\hline TCONS 00041960 & Upregulation & $\begin{array}{l}\text { Promotes osteogenic differentiation of } \\
\text { BMSCs }\end{array}$ & MiR-204-5p, miR-125a-3p & Shang et al., 2018 \\
\hline MSC-AS1 & Downregulation & Inhibits osteogenic differentiation of BMSCs & MiR-140-5p & Zhang et al., 2019c \\
\hline KCNQ1OT1 & Upregulation & $\begin{array}{l}\text { Stimulates osteogenic differentiation of } \\
\text { BMSCs }\end{array}$ & MiR-214 & Wang et al., 2019a \\
\hline TERC & Upregulation & Increases osteogenesis of hMSCs & MiR-217 & Gao et al., 2020a \\
\hline GAS5 & Upregulation & Promotes osteogenesis of hMSCs & MiR-498 & Feng et al., 2019 \\
\hline DGCR5 & Upregulation & Promotes osteogenesis of hMSCs & MiR-30d-5p & Wu et al., 2018c \\
\hline SNHG1 & Upregulation & Inhibits osteogenesis of BMSCs & Nedd4 and p-p38 & Huang et al., 2019a; Jiang et al., 2019b \\
\hline MEG3 & Upregulation & Inhibits osteogenesis of BMSCs & MiR-133a-5p & Wang et al., 2017a \\
\hline HoxA-AS3 & Downregulation & Increases MSCs osteogenesis & $\mathrm{EZH} 2$ & Zhu et al., 2016 \\
\hline MALAT1 & Downregulation & Inhibits osteogenic differentiation of BMSCs & MiR-143, MPK Pathway & Gao et al., 2018; Zheng et al., 2019 \\
\hline MALAT1 & Upregulation & $\begin{array}{l}\text { Promotes cell viability and survival in OB-6 } \\
\text { and hFOB1.19 cells }\end{array}$ & PPM1E & Fan et al., 2018 \\
\hline EPIC1 & Upregulation & $\begin{array}{l}\text { Stimulates cell survival of Dex-treated } \\
\text { osteoblasts }\end{array}$ & Myc & Zhang et al., 2018 \\
\hline ORLNC1 & Upregulation & $\begin{array}{l}\text { Increases the adipogenic differentiation of } \\
\text { BMSCs }\end{array}$ & MiR-296 & Yang et al., 2019 \\
\hline NEAT1 & Upregulation & $\begin{array}{l}\text { Promotes osteogenic differentiation of } \\
\text { hBMSCs }\end{array}$ & MiR-29b-3p & Zhang et al., 2019e \\
\hline XIXT & Downregulation & $\begin{array}{l}\text { Reduces osteogenic differentiation of } \\
\text { hBMSCs }\end{array}$ & MiR-30a-5p & Zhang et al., 2019b \\
\hline TINCR & Upregulation & Promotes migration of rat MSCs & MiR-761 & Zheng et al., 2020 \\
\hline MCF2L-AS1 & Upregulation & $\begin{array}{l}\text { Stimulates osteogenic differentiation in } \\
\text { BMSCs }\end{array}$ & MiR-33a & Chen et al., 2020 \\
\hline $\mathrm{H} 19$ & Downregulation & Inhibits osteogenic differentiation & MiR-188 & Wang et al., 2018a \\
\hline
\end{tabular}

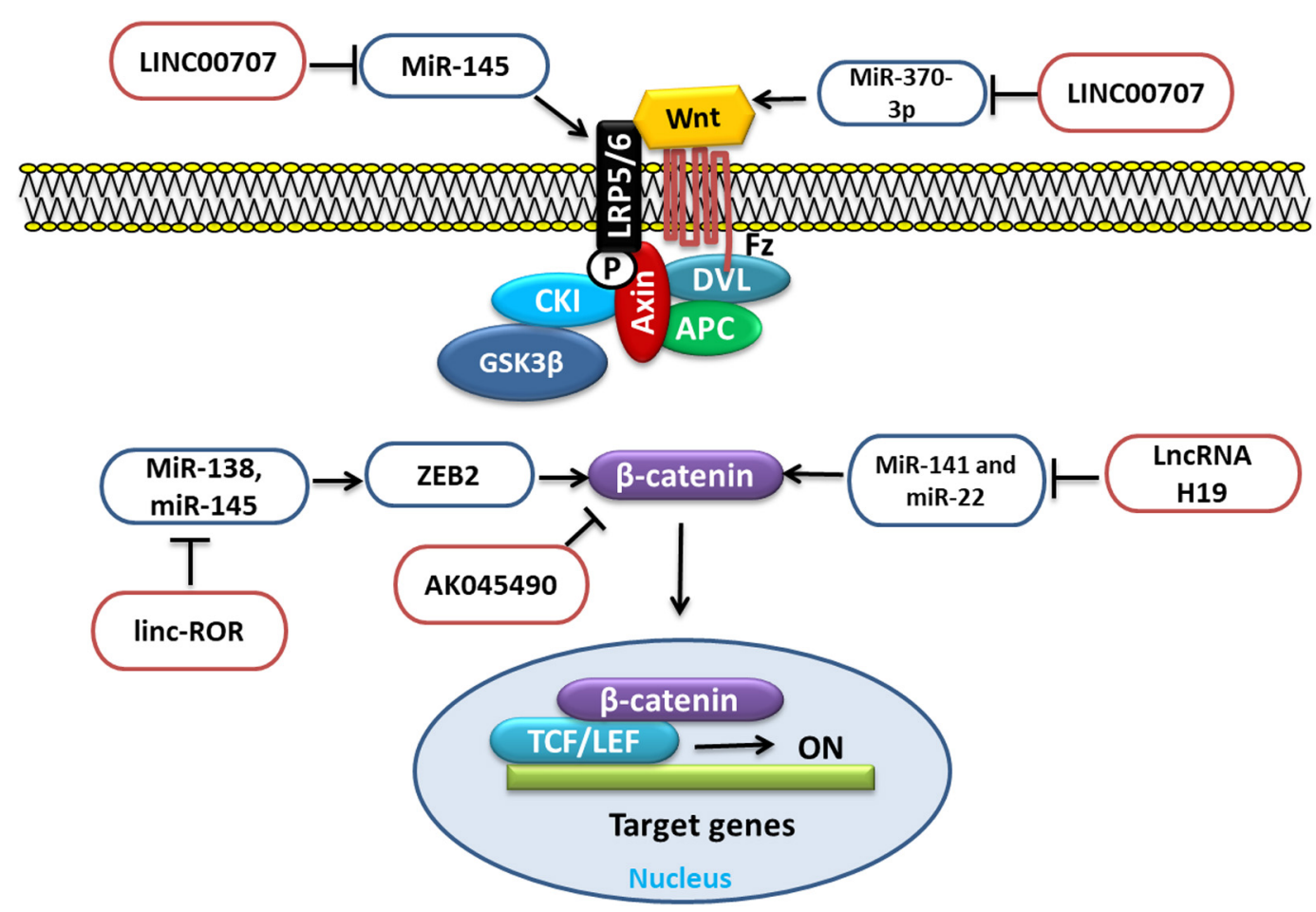

FIGURE 3 | LncRNA involved in BMSC regulation through Wnt signaling by targeting different miRNAs. 
TABLE 3 | LncRNAs involved in osteoclast regulation.

\begin{tabular}{|c|c|c|c|c|}
\hline LncRNA & Relative expression & Role & Target & References \\
\hline AK077216 & Upregulation & Increases formation and functions of osteoclasts & NFATc1 & Liu et al., 2019a \\
\hline MIRG & Upregulation & Stimulates osteoclastogenesis & MiR-1897 & Ling et al., 2019 \\
\hline CRNDE & Upregulation & Stimulates proliferation of osteoclasts & PI3K/Akt pathway & Li et al., 2018b \\
\hline LINC00311 & Upregulation & Promotes proliferation and the differentiation of osteoclasts & Delta-like 3 (DLL3) & Wang et al., 2018b \\
\hline TUG1 & Upregulation & Stimulates proliferation and reduces apoptosis in mice osteoclasts & PTEN & Han et al., 2019 \\
\hline CASC11 & Upregulation & Stimulates proliferation of osteoclasts & TNF- $\alpha$ & Yu et al., 2019 \\
\hline Bmncr & Upregulation & Lowers differentiation of osteoclasts and bone resorption & - & Chen et al., 2019a \\
\hline
\end{tabular}

"-" Unknown.

miR-22. These miRNAs negatively regulate the $\mathrm{Wnt} / \beta$-catenin pathway and osteogenesis. Thus, due to the repression of these two miRNAs, $\beta$-catenin could be derepressed, which eventually would stimulate the $\mathrm{Wnt} / \beta$-catenin pathway and osteogenesis (Liang et al., 2016). WNT2B, a member of the WNT family of proteins, which is an important player in various processes, such as cell proliferation and differentiation as well as osteogenic differentiation. It has been reported that, during osteogenic induction of hBMSCs, LncRNA LINC00707 overexpression promoted the osteogenic differentiation in vitro as well as in vivo by repressing miR-370-3p. This repression increased the WNT2B expression, which was the target of miR-370-3p (Jia et al., 2019). Low-density lipoprotein-related receptor 5 (LRP5) is an important receptor for Wnt signaling through which the signal is transduced, and the loss of LRP5 can increase the loss of bone mass (Williams, 2017). According to the study, the upregulation of LINC00707 could promote the $\mathrm{Wnt} / \beta$-catenin pathway by targeting miR-145. This regulates the expression of LRP5 and then activates the Wnt/ $\beta$-catenin pathway and increases the osteogenic differentiation of hBMSCs (Cai et al., 2020). Zinc finger E-box-binding homeobox 2 (ZEB2) is a member of the Zfh1 family. It regulates cell proliferation, invasion, and migration as well as apoptosis and also epithelialto-mesenchymal transition (EMT) in tumors. Importantly, it also regulates $\beta$-Catenin (Qi et al., 2012). MiR-138 and miR-145 through ZEB2 could negatively regulate osteogenesis. LncRNA linc-ROR upregulated during hBMSC osteogenic differentiation is shown to target miR-138 and miR-145. This antagonizing action of linc-ROR activated ZEB2, a target shared by these two miRNAs, which subsequently activated the Wnt/ $\beta$-catenin pathway and promoted osteogenesis (Feng et al., 2018a). Elevated lncRNA AK045490 is also reported to inhibit osteoblast differentiation of BMSCs, and its inhibition in vivo rescued bone formation in OVX osteoporosis mice. Furthermore, it is revealed that AK045490 targeted $\beta$-catenin and restricted its translocation into the nucleus, leading to suppression of Runx2, T-cell specific transcription factor (TCF1), and lymphoid enhancer binding factor (LEF1) expression and inhibition of osteoblast differentiation (Li et al., 2019a).

\section{LncRNAs in Osteoclast Regulation}

Osteoclast cells are derived from the monocyte-macrophage lineage and are responsible for resorbing and remodeling bone. In addition, osteoclasts also play an essential role as immune cells. Therefore, the molecular mechanisms involved in regulating not only the formation and functions of osteoclasts, but also their interactions with other cells can help understand their role in diseases (Boyce, 2013; Kylmaoja et al., 2016; Table 3).

According to Liu et al. (2019a), IncRNA AK077216 levels were associated with the regulation of different functions of osteoclasts. In vivo analysis in OVX mice has revealed the role of AK077216. It is demonstrated that the overexpression of AK077216 could increase the expression of the transcription factor, nuclear factor of activated T-cells 1 (NFATc1), whereas it could subdue NFAT-interacting protein 45 (NIP45) expression. This led to increased formation and functions of osteoclasts and, in turn, increased bone resorption (Liu et al., 2019a). Another lncRNA, lncRNA-MIRG, is reported to stimulate osteoclastogenesis when overexpressed. The study has further revealed that the MIRG functioned as a competitive endogenous RNA for miR-1897, which targeted NFATc1 and, thus, partially controlled the negative effect of miR-1897 on NFATc1 (Ling et al., 2019). LncRNA colorectal neoplasia differentially expressed (CRNDE) was also prominently expressed in osteoclasts of osteoporosis patients. The inhibition experiment has demonstrated the negative role of CRNDE on the cell cycle and cell proliferation and protein expressions of P53 although high expression of CRNDE stimulated the proliferation of osteoclasts through the PI3K/Akt signaling pathway (Li et al., 2018b). Another study has described the role of LncRNA LINC00311 in the Notch signaling pathway. Delta-like 3 (DLL3) is shown to be targeted by overexpressed LINC00311 and could lower the DLL3 expression. This downregulation could enhance cell proliferation by reducing apoptosis and differentiation by initiating the Notch signaling (Wang et al., 2018b). This promotes proliferation and, therefore, could increase the rate of bone resorption and enhance osteoporosis.

Long non-coding RNA taurine upregulated gene 1 (TUG1), which plays an effective role in the development of human cancers; ankylosing spondylitis, etc., is also shown to be involved in osteoporosis. Its upregulation in mice osteoclasts is reported to reduce the expression of PTEN and could stimulate proliferation and reduce apoptosis in mice osteoclasts (Han et al., 2019). Similarly, lncRNA cancer susceptibility 11 (CASC11), an oncogenic lncRNA that is identified in several types of cancer, such as gastric, colorectal, etc., has been found in abundance in osteoporotic patients. Further investigation has shown that, when IncRNA CASC11 was upregulated, it exercised a positive effect on the expression of TNF- $\alpha$ in osteoclast, which is known to activate osteoclasts and enhance 
bone resorption ( $Y u$ et al., 2019). Conversely, in the marrow and spleen of osteoporosis mice, Bmncr lncRNA was downregulated, but upregulation is shown to lower the expression of osteoclastmarker genes, such as Atp6v0d2, Acp5, Ctr, and Mmp9, and differentiation of osteoclasts induced by RANKL. Moreover, it also reduced the ability of bone resorption, thus reducing osteoporosis progression (Chen et al., 2019a).

\section{LncRNAs in Osteoblast Regulation}

Osteoblasts are primarily involved in the formation of bone through different pathways, such as the PI3K/Akt cell signaling pathway, MAPK pathway, $\mathrm{Wnt} / \beta$-catenin signaling, etc. The PI3K/Akt cell signaling pathway plays an important role in inhibiting osteoporosis by stimulating proliferation, differentiation of osteoblasts, and bone formation (Xi et al., 2015). In osteoblasts of PMOP rats, lower levels of phosphoPI3K (p-PI3K), p-Akt, and p-phosphoinositide-dependent kinase-1(PDK1) have been reported to be regulated by lncRNA AK023948 (AK0). It is also shown that the phosphorylation level of AKT was increased in the osteoblasts overexpressing the AK0 gene, and downregulation and knocking out decreased AKT phosphorylation levels in osteoblasts (Wang et al., 2020b). LncRNA AK125437, on the other hand, is reported to regulate the osteoblasts through the MAPK pathway to influence the bone mineral density in postmenopausal osteoporosis rats through p-p38 (Wang et al., 2020a). For osteoblast differentiation, lncRNA, antidifferentiation ncRNA (ANCR) is described as an indispensable factor. ANCR upregulation is shown to inhibit not only proliferation, but also osteogenesis of PMOP osteoblast cells in vitro and osteoid formation in vivo. This inhibition is credited to the lowered expression of Runx2 by the association of lncRNA-ANCR and enhancer of zeste homolog 2 (EZH2; Cai et al., 2019), whereas the elevated expression of lncRNA UCA1 in the plasma of osteoporosis patients inhibited osteoblast function. The inhibition of UCA1 showed increased proliferation and differentiation of preosteoblast MC3T3E1 cell lines by stimulating bone morphogenetic protein-2 (BMP-2)/(Smad1/5/8) signaling pathway (Zhang et al., 2019d). Some IncRNAs are also expressed under the influence of microgravity. One such example is osteoblast differentiationrelated IncRNA under simulated microgravity (ODSM). ODSM is reported to prevent the apoptosis of osteoblasts and enhance osteoblast mineralization in vitro (Wang et al., 2020c). Furthermore, it is described that the apoptosis in MC3T3-E1 could be reduced cells, and differentiation could be promoted in MC3T3-E1 cells subject to microgravity by the increasing level of lncRNA ODSM. In addition, the supplementation of LncRNA ODSM in mice under microgravity conditions reduced the apoptosis in bone tissue and increased osteoblast activity (Wang et al., 2020c).

According to Liu et al., during osteoblast differentiation, lncRNA TUG1 as well as marker genes, such as Axin 2, Frizzled-2, Runx 2 , and $\beta$-catenin, were upregulated. It is concluded that the silencing of lncRNA TUG1 using short hairpin TUG1 (shTUG1) could lower the OCN, OPN, and ALP activity and proliferation capability of osteoblast in addition to marker genes (Liu et al., 2019c). Similarly, IncRNA, differentiation antagonizing non-protein coding RNA (DANCR) could also regulate the proliferation and differentiation of preosteoblast cells through Wnt/ $\beta$-catenin signaling (Jiang et al., 2019a). In vitro experiments revealed the inhibition of DANCR was able to stimulate the expression of differentiation-related genes, such as Runx2, collagen type I alpha 1, OSX and mineralization. Moreover, DANCR could also promote $\beta$-catenin expression, thus activating the $\mathrm{Wnt} / \beta$-catenin pathway and promoting preosteoblast cells to proliferate and differentiate into osteoblasts (Jiang et al., 2019a). LncRNA MEG3 silencing is also reported to stimulate preosteoblast MC3T3-E1 cells to proliferate and differentiate into osteoblasts. The inhibition showed increased levels of $W n t$ and $\beta$-catenin proteins, suggesting increased $W n t / \beta$-catenin signaling in osteoblasts (Li et al., 2019b). In bone formation, mineralization of the matrix is an important step, which is secreted by osteoblasts. In such cells, according to the study, IncRNA H19 was upregulated. Subsequent investigations showed H19 could promote osteoblast mineralization by targeting miR-185-5p, which inhibited IGF1 expression, an important factor involved in matrix mineralization (Wu et al., 2019; Table 4).

In summary, IncRNAs play a significant role in bone metabolism and osteoporosis. Through different pathways, IncRNAs influence different aspects of bone cells, such as proliferation, differentiation, and survival, indicating their potential in pharmaceutical therapeutics.

\section{CircRNA}

Circular RNA is another group of endogenous ncRNAs that are circular, were originally considered to be by-products of abnormal splicing, and have a transcript length of hundreds to thousands of nucleotides. The common circRNAs that have been discovered to date originate from various segments of a gene, such as exons in the coding region of a gene, $5^{\prime}$ - or $3^{\prime}$-untranslated regions (UTRs), introns, and regions between two genes, antisense RNAs, etc (Huang et al., 2019c; Lei et al., 2019). The lack of a $5^{\prime}$ or $3^{\prime}$ end in circRNAs confers them the stability and resistance to exonuclease digestion (Bose and Ain, 2018). CircRNAs are exclusively expressed in the cytoplasm and among diverse species and are highly conserved. CircRNAs have a great many functions in cellular activities. They act as an miRNA sponge and regulate transcription as well as protein and peptide coding (Zhai et al., 2018). They are also implicated in various diseases, such as cancer, bone diseases, etc. Because of their high stability and tissue specificity as well as disease-specific expression, circRNAs function as potential biomarkers for both diseases and prognosis (Zhai et al., 2018).

Circular RNAs are covalently closed-loop structures that are formed through backsplicing, a type of alternative splicing (Ebbesen et al., 2017). Pre-mRNA can generate a linear or circular RNA. If pre-mRNA goes through canonical splicing, it leads to the formation of exon-included linear RNA, and if it undergoes backsplicing, it produces circular RNA. Both canonical splicing signals as well as spliceosomal machinery 
TABLE 4 | LncRNAs involved in osteoblast regulation.

\begin{tabular}{|c|c|c|c|c|}
\hline LncRNA & Relative expression & Role & Target & References \\
\hline AK023948 & Downregulation & Reduces proliferation, differentiation of osteoblast & AKT & Wang et al., 2020b \\
\hline ANCR & Upregulation & Inhibits the proliferation and osteogenesis of PMOP osteoblast & RUNX2 & Cai et al., 2019 \\
\hline UCA1 & Downregulation & $\begin{array}{l}\text { Increases proliferation and differentiation of osteoblast MC3T3-E1 } \\
\text { cells }\end{array}$ & BMP-2/Smad1/5/8 pathway & Zhang et al., 2019d \\
\hline ODSM & Upregulation & $\begin{array}{l}\text { Prevents apoptosis of osteoblasts and enhance osteoblast } \\
\text { mineralization }\end{array}$ & - & Wang et al., 2020c \\
\hline TUG1 & Upregulation & Increases osteoblast differentiation & - & Liu et al., 2019c \\
\hline DANCR & Downregulation & $\begin{array}{l}\text { Promotes preosteoblast proliferation and differentiation into } \\
\text { osteoblasts. }\end{array}$ & $\beta$-catenin & Jiang et al., 2019a \\
\hline MEG3 & Downregulation & $\begin{array}{l}\text { Stimulates preosteoblasts MC3T3-E1 cells to proliferate and } \\
\text { differentiate into osteoblast }\end{array}$ & Wnt and $\beta$-catenin & Li et al., 2019b \\
\hline $\mathrm{H} 19$ & Upregulation & Promotes osteoblast mineralization & MiR-185-5p & Wu et al., 2019 \\
\hline
\end{tabular}

"-" Unknown.

are essentially backspliced exon circularization (Chen and Yang, 2015). CircRNA formation is generally classified into two mechanisms, namely direct backsplicing and lariat intermediate or exon skipping (Jeck and Sharpless, 2014). In backsplicing, intervening introns and two unspliced introns within a transcript pair are spliced. The donor of the $3^{\prime}$ intron is attacked by a branch point in the $5^{\prime}$ intron and the backsplice is completed by the $3^{\prime}$ donor through attacking the $5^{\prime}$ acceptor, forming a circular RNA, whereas, in exon skipping, it forms a lariat containing an exon that is spliced to remove the intronic sequence, forming a circular RNA (Jeck and Sharpless, 2014).

\section{Circular RNA Involved in BMSC Regulation}

Some circRNAs inhibit osteogenic differentiation or promote adipogenesis of BMSCs/AMSCs, and others promote osteogenesis or inhibit the adipogenesis of BMSCs/AMSCs (Figure 4). Circular RNAs involved in BMSC regulation include hsa-circRNA 0074834, hsa-circRNA 0006393, circRNAs POMT1 and MCM3AP, circRNAs RFWD2 and INO80, circRNA 0076906, circRNA 0016624, circRNA 0048211, etc.

Bone fractures have the ability to repair themselves, but some bone fractures fail to heal, and in about $5 \%$ of cases, it leads to non-unions (Leighton et al., 2017). In such cases, the MSC-osteoblast lineage plays a significant role (Gibon et al., 2016). Some circRNAs help BMSCs toward osteogenesis, such as hsa-circ 0074834, which is reported to promote osteogenic differentiation of BMSCs and restore bone defects. The level of hsa-circRNA 0074834 in BMSCs from patients with bone fractures is reported to be decreased, and upregulation could target miR-942-5p to control the expression of E-Box binding homeobox 1 (ZEB1) and vascular endothelial growth factor (VEGF; Ouyang et al., 2019).

In BMSCs from GIOP patients, the downregulated expression of hsa-circRNA 0006393 has been reported. It is shown that hsa-circRNA 0006393 targeted miR-145-5p and also upregulated FOXO1 to increase the expression level of osteogenesis-associated genes (Wang et al., 2019b). Similarly, low expression of circRNAs POMT1 and MCM3AP is shown during hAMSc osteogenic differentiation. The loss-of-function experiment has revealed circRNA POMT1 and MCM3AP inhibition increased the expression of Runx2 and COL I. Furthermore, the study also showed increased expression of hsa-miR-6881-3p, which inhibited Smad6 and Chordin, the well-known inhibitors of BMP signaling. Thus, it can be concluded that the circRNAs POMT1 and MCM3AP could promote osteogenesis through BMPs signaling (Huang et al., 2019d). Another study has also reported the low levels of circRNA 0016624 during PMOP. The study resulted in establishing that the upregulation of circRNA 0016624 could induce BMP2 expression by acting as ceRNA to regulate the expression of miR-98, which previously has described targeting BMP2. Thus, circRNA 0016624 regulated the expression of miR-98 to increase osteogenic differentiation in vitro (Yu and Liu, 2019).

The bone formation and healing of bone defects can be promoted by a nel-like molecule, type 1 (NELL-1). The study conducted to investigate the effect of NELL-1 on the induction of osteogenesis in hASCs describes the high levels of circRNAs RFWD2 and circINO80 in hASCs. The inhibition of circRNAs RFWD2 and circINO80 showed reduced osteogenesis. Further investigation discovered that circRNAs RFWD2 and circINO80 interacted with hsa-miR-6817-5p, a negative regulator of osteogenesis. Thus, by sponging the action of has-miR-6817, circRNAs RFWD2 and circINO80 promoted osteogenesis (Huang et al., 2019b). Additionally, increased circRNA 0076906 expression during osteogenic differentiation of hMSCs is also shown to positively regulate osteogenesis. This positive regulation resulted from the sponging action of miR-1305, which targeted osteoglycin (OGN) to regulate osteogenic differentiation through the miR1305/OGN pathway (Wen et al., 2020). Similarly, in another study, hBMSCs collected from PMOP patients undergoing osteogenic differentiation have shown upregulated circRNA 0048211, which also increased the expression of OCN, RUNX2, OPN, and ALP activity. Furthermore, the study has shown that circRNA 0048211 targeted miRNA-93-5p and consequently enhanced BMP2 expression (Qiao et al., 2020; Table 5). 


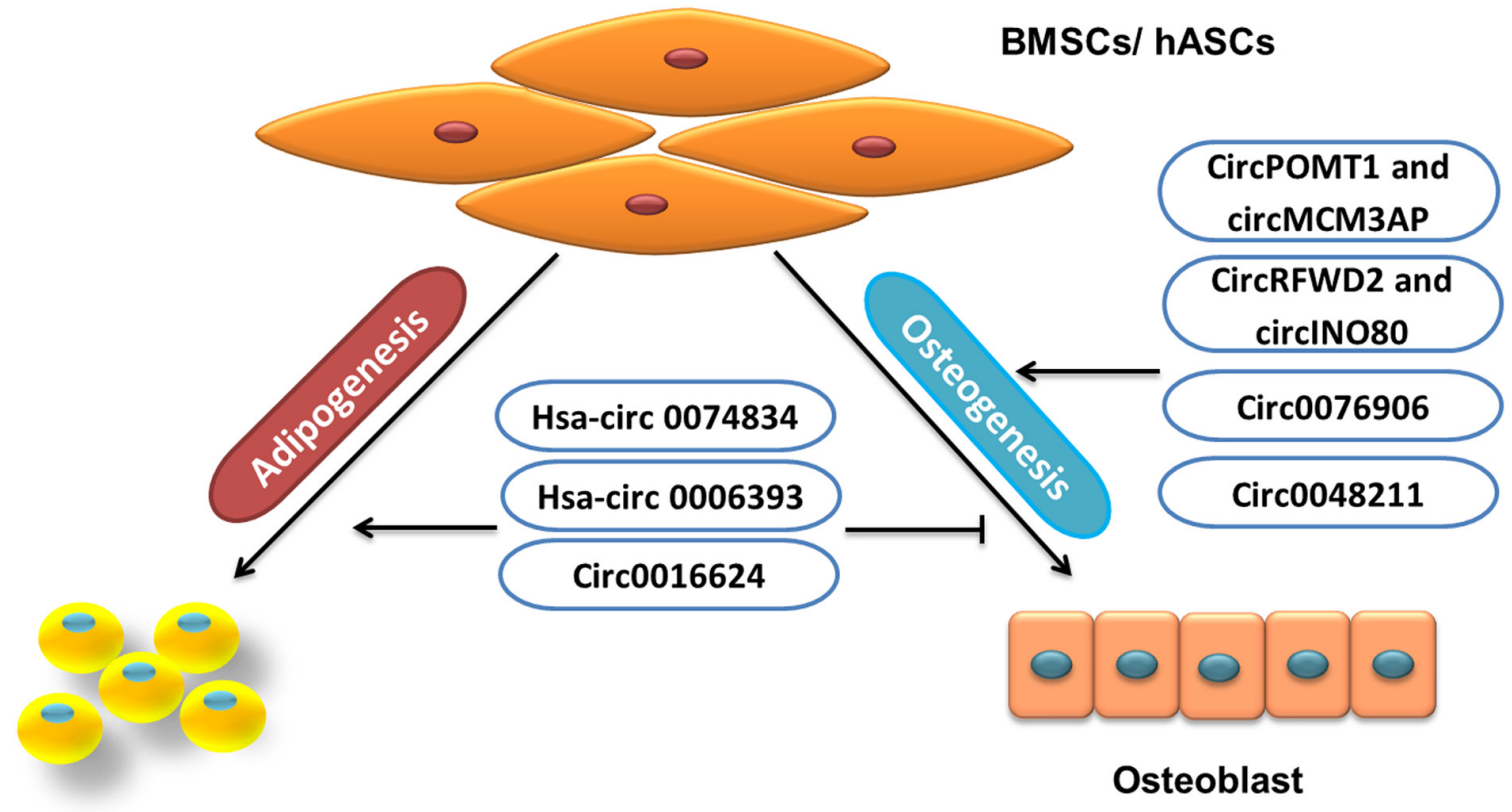

Adipocytes

FIGURE 4 | Role of circRNA in BMSCs/hASCs fate.

TABLE 5 | Role of circRNAs in BMSCs.

\begin{tabular}{|c|c|c|c|c|}
\hline CircRNA & Relative expression & Role & Target & References \\
\hline 0074834 & Upregulation & Inhibits osteogenic differentiation of BMSCs & MiR-942-5p & Ouyang et al., 2019 \\
\hline 0006393 & Upregulation & Inhibits osteogenesis & MiR-145-5p & Wang et al., 2019b \\
\hline POMT1 and MCM3AP & Downregulation & Promotes osteogenesis of hASCs & Hsa-miR-6881-3p & Huang et al., 2019d \\
\hline 0016624 & Upregulation & Inhibits osteogenesis & MiR-98 & Yu and Liu, 2019 \\
\hline RFWD2 and INO80 & Upregulation & Promotes osteogenesis of hASCs & Hsa-miR-6817-5p, & Huang et al., 2019c \\
\hline 0076906 & Upregulation & Promotes osteogenic differentiation of hMSCs & MiR-1305 & Wen et al., 2020 \\
\hline 0048211 & Upregulation & Increases osteogenic differentiation of hBMCs & MiR-93-5p & Qiao et al., 2020 \\
\hline
\end{tabular}

TABLE 6 | Role and target of circRNAs in osteoblasts.

\begin{tabular}{|c|c|c|c|c|}
\hline CircRNA & Relative expression & Role & Target & References \\
\hline 003795 & Upregulation & Promotes osteoblast differentiation and mineralization & COL15A1 & Wu et al., 2020 \\
\hline AFF4 & Upregulation & Promotes MC3T3-E1 proliferation & MiR-7223-5p & Mi et al., 2019 \\
\hline HIPK3 & Upregulation & Increases cell viability in OB-6 osteoblastic and primary human osteoblast cells & MiR-124 & Liang et al., 2020 \\
\hline cPWWP2A & Upregulation & Inhibits apoptosis in osteoblast & MiR-579, & Hong et al., 2019 \\
\hline
\end{tabular}

\section{CircRNA Involved in Osteoclast Regulation}

In bone marrow monocyte/macrophage (BMM) cells, the elevated expression of circRNA 28313 indicates when cells were induced. When circRNA 28313 was knocked down in vitro, differentiation of osteoclasts was repressed. In addition, it also inhibited bone resorption in OVX mice (Chen et al., 2019b). The study additionally employed the bioinformatics tool to further investigate the role of circRNA 20313. It demonstrated that circRNA 28313 and CSF1 acted as a binding site for miR-195a and collectively formed a circRNA-miRNAmRNA network. Furthermore, it showed that circRNA 28313 functioned as ceRNA in targeting miR-195a to promote CSF1 and to regulate the osteoclast differentiation in BMM cells (Chen et al., 2019b).

\section{CircRNA Involved in Osteoblast Regulation}

In osteoblasts and stromal cells, collagen type XV (ColXV), a bone extracellular matrix (ECM) protein helps to maintain 
potency and to promote differentiation (Lisignoli et al., 2017). The study has described that differentiation-induced MC3T3E1 and MDPC23 osteoblasts exhibited increased mmu-circRNA 003795 and OPN expression. It further reports that when mmu-circ003795 was inhibited, expression levels of COL15A1 and OPN were decreased but that of miR-1249-5p was elevated. Thus, it indicates that, through miR-1249-5p, mmucircRNA 003795 could target COL15A1 to regulate osteoblast differentiation and mineralization (Wu et al., 2020). For the activation of PI3K/Akt signaling and the phosphorylation of Akt, class I PI3K regulatory subunit $1(\alpha)$ (PIK3R1) is required (Cui et al., 2016), which is reported to be increased during fracture healing. The study suggests the PIK3R1 expression was proportional to the increased proliferation and lowered apoptosis of MC3T3-E1 cells. Moreover, in these cells, circRNA AFF4 silencing is shown to reduce $\mathrm{Bcl}-2$ expression, an antiapoptotic protein, as well as PIK3R1. Furthermore, it showed circRNA AFF4 targeted miR-7223-5p, which inhibited PI3KR1, thus confirming circRNA AFF4 as a positive regulator of MC3T3-E1 proliferation (Mi et al., 2019). It is well known that reactive oxygen species (ROS) can kill bacteria as well as human cells. ROS also play an important role in diseases. It is not only the excessive amount that can lead to diseases, but a lack of ROS also contributes to the pathogenesis of diseases (Brieger et al., 2012). Reactive oxygen species, such as superoxide and hydrogen peroxide, significantly contribute to the progression of skeletal aging and various bone diseases (Callaway and Jiang, 2015). In hydrogen peroxidetreated human osteoblasts, reduced homeodomain-interacting protein kinase 3 (HIPK3) lncRNA expression was reported by Liang et al. (2020). The study demonstrated that the lentivirus-mediated circHIPK3 overexpression could increase cell viability in OB-6 osteoblastic and primary human osteoblast cells by inhibiting cell apoptosis. Furthermore, circHIPK3 silencing stimulated miR-124, which targeted cyclin-dependent kinase 6 and Rho-associated Protein Kinase 1 to increase cytotoxicity in OB-6 cells and primary human osteoblasts (Liang et al., 2020). In another study, the analysis of Dexinduced human osteoblasts and tissues of the necrotic femoral head of Dex-taking patients showed decreased cPWWP2A circRNA levels, but overexpression of cPWWP2A is shown to inhibit the miR-579, increasing the expression of SIRT1 and PDK1 (phosphoinositide-dependent protein kinase 1), the targets of miR-579 and cell death and apoptosis. Thus, by sponging, the actions of miR-579 cPWWP2A are shown to attenuate cytotoxicity in human osteoblasts (Hong et al., 2019; Table 6).

\section{CONCLUSION AND PERSPECTIVE}

In this article, we have reviewed the different roles of lncRNAs and circRNAs in different bone cells and osteoporosis. The different lncRNAs and circRNAs in the serum of diseased patients as well as different bone cells can act as biomarkers. Many lncRNAs and circRNAs also act as competitive endogenous RNAs for many microRNAs to regulate the expression of microRNA targeted genes affecting different pathways that are important in the regulation of BMSCs, osteoblasts, osteoclasts, and osteogenesis. Some lncRNA and circRNAs that are upregulated in disease can be targeted to inhibit their expression although those that are downregulated can be stimulated to express sufficiently to increase bone formation.

Due to their abundance and ability to influence the different aspects of bone metabolism, lncRNAs and circRNAs have been shown as biomarkers in various diseases. Many reports mention lncRNAs, such as MALAT1 and SOX2-OT as potential biomarkers in various bone diseases, such as osteosarcoma (Wang et al., 2017b; Liu et al., 2019b) and lncRNA SNHG5, and CRNDE in acute myeloid leukemia (Li and Sun, 2018; Wang et al., 2018c), etc. Some reports mention exosomes that are rich in circRNA as a promising biomarker for diagnosing cancers (Li et al., 2015), such as lung adenocarcinoma (Liu et al., 2019d), circRNA 0004277 in acute myeloid leukemia (Li et al., 2017), circRNA NT5C2 in osteosarcoma (Nie et al., 2018), cardiovascular disease, etc. Because of the high-throughput techniques, such as Chip Scanning Analysis, it has become possible to detect the expression profile of different circRNA in various diseases, such as osteoarthritis and Kashin-Beck disease (Wang et al., 2020d).

New therapeutics could also be developed by targeting these ncRNAs. Some reports suggest the use of ASO or siRNAs, miRNAs, and circRNAs in different diseases, such as cardiovascular or cancers (Holdt et al., 2018; Lucas et al., 2018; Gao et al., 2020b). The major problem that hinders the use of ncRNAs, including lncRNAs and circRNAs, as potential therapeutics is their stability and delivery to the target cells, but advances in chemical modifications and strategies have proved that delivery systems can be improved to reduce unwanted side effects (Patil et al., 2019).

Still, the fact remains that there is a lack of detailed study on circRNAs in osteoporosis. Therefore, future research in this area could assuredly help understand bone metabolism and osteoporosis. Furthermore, undoubtedly much research is required to uncover the potential of lncRNAs and circRNAs in clinical settings in developing therapeutics to treat not only bone-related diseases, but also other life-threatening diseases.

\section{AUTHOR CONTRIBUTIONS}

SP and YG: writing - original draft preparation. SP, KD, XZ, YG, and AQ: writing - review and editing. AQ: funding acquisition.

\section{FUNDING}

The authors gratefully acknowledge the financial assistance provided by the Shaanxi Provincial Science and Technology Department (2019JM-230 and 2020JM-100), National Natural Science Foundation of China (81700784 and 82072106) and the grant BK 17J004. 


\section{REFERENCES}

Adami, G., and Saag, K. G. (2019). Glucocorticoid-induced osteoporosis: 2019 concise clinical review. Osteoporos Int. 30, 1145-1156. doi: 10.1007/s00198019-04906-x

Armas, L. A., and Recker, R. R. (2012). Pathophysiology of osteoporosis: new mechanistic insights. Endocrinol. Metab. Clin. North Am. 41, 475-486. doi: 10.1016/j.ecl.2012.04.006

Arrigoni, A., Ranzani, V., Rossetti, G., Panzeri, I., Abrignani, S., Bonnal, R. J. P., et al. (2016). Analysis RNA-seq and Noncoding RNA. Methods Mole. Biol 1480, 125-135. doi: 10.1007/978-1-4939-6380-5_11

Asharani, P. V., Keupp, K., Semler, O., Wang, W., Li, Y., and Thiele, H. (2012). Attenuated BMP1 function compromises osteogenesis, leading to bone fragility in humans and zebrafish. Am. J. Hum. Genet 90, 661-674. doi: 10.1016/j.ajhg. 2012.02.026

Baker, N., Boyette, L. B., and Tuan, R. S. (2015). Characterization of bone marrowderived mesenchymal stem cells in aging. Bone 70, 37-47. doi: 10.1016/j.bone. 2014.10.014

Bose, R., and Ain, R. (2018). Regulation of Transcription by Circular RNAs. Adv. Exp. Med. Biol. 1087, 81-94. doi: 10.1007/978-981-13-1426-1_7

Boyce, B. F. (2013). Advances in the regulation of osteoclasts and osteoclast functions. J. Dent Res. 92, 860-867. doi: 10.1177/0022034513500306

Brieger, K., Schiavone, S., Miller, F. J. Jr., and Krause, K. H. (2012). Reactive oxygen species: from health to disease. Swiss Med. Wkly 142:w13659. doi: 10.4414/smw. 2012.13659

Cai, N., Li, C., and Wang, F. (2019). Silencing of LncRNA-ANCR Promotes the Osteogenesis of Osteoblast Cells in Postmenopausal Osteoporosis via Targeting EZH2 and RUNX2. Yonsei Med. J. 60, 751-759. doi: 10.3349/ymj.2019.60.8.751

Cai, W. L., Zeng, W., Liu, H. H., Zhu, B. Y., Liu, J. L., and Liu, Y. (2020). LncRNA LINC00707 promotes osteogenic differentiation of hBMSCs through the Wnt/ $\beta$-catenin pathway activated by LINC00707/miR-145/LRP5 axis. Eur. Rev. Med. Pharmacol. Sci. 24, 18-28.

Callaway, D. A., and Jiang, J. X. (2015). Reactive oxygen species and oxidative stress in osteoclastogenesis, skeletal aging and bone diseases. J. Bone Miner. Metab. 33, 359-370. doi: 10.1007/s00774-015-0656-4

Chen, L.-L., and Yang, L. (2015). Regulation of circRNA biogenesis. RNA Biol. 12, 381-388. doi: 10.1080/15476286.2015.1020271

Chen, Q., Wang, M., and Wu, S. (2020). The lncRNA MCF2L-AS1 controls osteogenic differentiation by regulating miR-33a. Cell Cycle 19, 1059-1065 doi: 10.1080/15384101.2020.1747776

Chen, R. S., Zhang, X. B., Zhu, X. T., and Wang, C. S. (2019a). LncRNA Bmncr alleviates the progression of osteoporosis by inhibiting RANML-induced osteoclast differentiation. Eur. Rev. Med. Pharmacol. Sci. 23, 9199-9206.

Chen, X., Ouyang, Z., Shen, Y., Liu, B., Zhang, Q., Wan, L., et al. (2019b). CircRNA_28313/miR-195a/CSF1 axis modulates osteoclast differentiation to affect OVX-induced bone absorption in mice. RNA Biol. 16, 1249-1262. doi: $10.1080 / 15476286.2019 .1624470$

Chen, X., Yang, L., Ge, D., Wang, W., Yin, Z., Yan, J., et al. (2019c). Long noncoding RNA XIST promotes osteoporosis through inhibiting bone marrow mesenchymal stem cell differentiation. Exp. Ther. Med. 17, 803-811. doi: 10 3892/etm.2018.7033

Chen, X., Yang, T., Wang, W., Xi, W., Zhang, T., Li, Q., et al. (2019d). Circular RNAs in immune responses and immune diseases. Theranostics 9, 588-607. doi: 10.7150/thno. 29678

Chi, Y., Wang, D., Wang, J., Yu, W., and Yang, J. (2019). Long Non-Coding RNA in the Pathogenesis of Cancers. Cells 8:1015. doi: 10.3390/cells8091015

Cui, X., Wang, S., Cai, H., Lin, Y., Zheng, X., Zhang, B., et al. (2016). Overexpression of microRNA-634 suppresses survival and matrix synthesis of human osteoarthritis chondrocytes by targeting PIK3R1. Sci. Rep. 6:23117. doi: $10.1038 /$ srep23117

Ding, S., Zhu, Y., Liang, Y., Huang, H., Xu, Y., and Zhong, C. (2018). Circular RNAs in Vascular Functions and Diseases. Adv. Exp. Med. Biol. 1087, 287-297. doi: 10.1007/978-981-13-1426-1_23

Dumitrescu, A. M., Di Cosmo, C., Liao, X. H., Weiss, R. E., and Refetoff, S. (2010). The syndrome of inherited partial SBP2 deficiency in humans. Ant. Redox. Sign. 12, 905-920. doi: 10.1089/ars.2009.2892

Ebbesen, K. K., Hansen, T. B., and Kjems, J. (2017). Insights into circular RNA biology. RNA Biol. 14, 1035-1045. doi: 10.1080/15476286.2016.1271524
Eiken, P., and Vestergaard, P. (2016). Treatment of osteoporosis after alendronate or risedronate. Osteoporos Int. 27, 1-12. doi: 10.1007/s00198-015-3334-4

Esteller, M. (2011). Non-coding RNAs in human disease. Nat. Rev. Genet. 12, 861-874. doi: 10.1038/nrg3074

Fan, J., Zhang, Y., Liu, W., Zhu, X., Xu, D., Zhao, J., et al. (2018). Long Non-Coding RNA MALAT1 Protects Human Osteoblasts from Dexamethasone-Induced Injury via Activation of PPM1E-AMPK Signaling. Cell. Physiol. Biochem. 51, 31-45. doi: 10.1159/000495159

Feng, J., Wang, J. X., and Li, C. H. (2019). LncRNA GAS5 overexpression alleviates the development of osteoporosis through promoting osteogenic differentiation of MSCs via targeting microRNA-498 to regulate RUNX2. Eur. Rev. Med. Pharmacol. Sci. 23, 7757-7765.

Feng, L., Shi, L., Lu, Y.-F., Wang, B., Tang, T., Fu, W.-M., et al. (2018a). LincROR Promotes Osteogenic Differentiation of Mesenchymal Stem Cells by Functioning as a Competing Endogenous RNA for miR-138 and miR-145. Mole. Ther. Nucleic Acids 11, 345-353. doi: 10.1016/j.omtn.2018.03.004

Feng, X., Lin, T., Liu, X., Yang, C., Yang, S., and Fu, D. (2018b). Long non-coding RNA BDNF-AS modulates osteogenic differentiation of bone marrow-derived mesenchymal stem cells. Mol. Cell Biochem. 445, 59-65. doi: 10.1007/s11010017-3251-2

Gao, G. C., Yang, D. W., and Liu, W. (2020a). LncRNA TERC alleviates the progression of osteoporosis by absorbing miRNA-217 to upregulate RUNX2. Eur. Rev. Med. Pharmacol. Sci. 24, 526-534.

Gao, Y., Patil, S., and Qian, A. (2020b). The Role of MicroRNAs in Bone Metabolism and Disease. Int. J. Mole. Sci. 21:6081. doi: 10.3390/ijms21176081

Gao, Y., Xiao, F., Wang, C., Wang, C., Cui, P., Zhang, X., et al. (2018). Long noncoding RNA MALAT1 promotes osterix expression to regulate osteogenic differentiation by targeting miRNA-143 in human bone marrow-derived mesenchymal stem cells. J. Cell. Biochem. 119, 6986-6996. doi: 10.1002/jcb. 26907

Ghafouri-Fard, S., Esmaeili, M., and Taheri, M. (2020). H19 lncRNA: Roles in tumorigenesis. Biomed. Pharmacother. 123:109774. doi: 10.1016/j.biopha.2019. 109774

Gibon, E., Lu, L., and Goodman, S. B. (2016). Aging, inflammation, stem cells, and bone healing. Stem Cell Res. Ther. 7:44. doi: 10.1186/s13287-016-0300-9

Han, Y., Liu, C., Lei, M., Sun, S., Zheng, W., Niu, Y., et al. (2019). LncRNA TUG1 was upregulated in osteoporosis and regulates the proliferation and apoptosis of osteoclasts. J. Orthop. Surg. Res. 14, 416-416. doi: 10.1186/s13018-019-1430-4

Holdt, L. M., Kohlmaier, A., and Teupser, D. (2018). Circular RNAs as Therapeutic Agents and Targets. Front. Physiol. 9, 1262-1262. doi: 10.3389/fphys.2018.01262

Hong, H., Sun, Y., Deng, H., Yuan, K., Chen, J., Liu, W., et al. (2019). Dysregulation of cPWWP2A-miR-579 axis mediates dexamethasone-induced cytotoxicity in human osteoblasts. Biochem. Biophys. Res. Commun. 517, 491-498. doi: 10. 1016/j.bbrc.2019.07.095

Huang, S., Zhu, X., Xiao, D., Zhuang, J., Liang, G., Liang, C., et al. (2019a). LncRNA SNHG1 was down-regulated after menopause and participates in postmenopausal osteoporosis. Biosci. Rep. 39:BSR20190445. doi: 10.1042/ BSR20190445

Huang, T., Alvarez, A., Hu, B., and Cheng, S. Y. (2013). Noncoding RNAs in cancer and cancer stem cells. Chin. J. Cancer 32, 582-593. doi: 10.5732/cjc.013.10170

Huang, X. Q., Cen, X., Sun, W. T., Xia, K., Yu, L. Y., Liu, J., et al. (2019b). CircPOMT1 and circMCM3AP inhibit osteogenic differentiation of human adipose-derived stem cells by targeting miR-6881-3p. Am. J. Transl. Res. 11, 4776-4788.

Huang, X., Cen, X., Zhang, B., Liao, Y., Zhao, Z., Zhu, G., et al. (2019c). The roles of circRFWD2 and circINO80 during NELL-1-induced osteogenesis. J. Cell Mol. Med. 23, 8432-8441. doi: $10.1111 / \mathrm{jcmm} .14726$

Huang, X., Cen, X., Zhang, B., Liao, Y., Zhu, G., Liu, J., et al. (2019d). Prospect of circular RNA in osteogenesis: A novel orchestrator of signaling pathways. J. Cell Physiol. 234, 21450-21459. doi: 10.1002/jcp.28866

Huang, Y., Zheng, Y., Jia, L., and Li, W. (2015). Long Noncoding RNA H19 Promotes Osteoblast Differentiation Via TGF- $\beta 1 /$ Smad3/HDAC Signaling Pathway by Deriving miR-675. Stem Cells 33, 3481-3492. doi: 10.1002/stem. 2225

Jeck, W. R., and Sharpless, N. E. (2014). Detecting and characterizing circular RNAs. Nat. Biotechnol. 32, 453-461. doi: 10.1038/nbt.2890

Jeon, S. A., Lee, J. H., Kim, D. W., and Cho, J. Y. (2018). E3-ubiquitin ligase NEDD4 enhances bone formation by removing TGF $\beta 1$-induced pSMAD1 
in immature osteoblast. Bone 116, 248-258. doi: 10.1016/j.bone.2018. 08.012

Jia, B., Wang, Z., Sun, X., Chen, J., Zhao, J., and Qiu, X. (2019). Long noncoding RNA LINC00707 sponges miR-370-3p to promote osteogenesis of human bone marrow-derived mesenchymal stem cells through upregulating WNT2B. Stem Cell Res. Ther. 10, 67-67. doi: 10.1186/s13287-019-1161-9

Jiang, S. Y., Miao, Y. X., Hirokazu, T., Zhu, S. Z., and Lu, J. S. (2019a). Effects of lncRNA DANCR on proliferation and differentiation of osteoblasts by regulating the Wnt/ $\beta$-catenin pathway. Eur. Rev. Med. Pharmacol. Sci. 23, 5558-5566.

Jiang, Y., Wu, W., Jiao, G., Chen, Y., and Liu, H. (2019b). LncRNA SNHG1 modulates p38 MAPK pathway through Nedd4 and thus inhibits osteogenic differentiation of bone marrow mesenchymal stem cells. Life sci. 228, 208-214. doi: 10.1016/j.lfs.2019.05.002

Ju, W., Luo, X., and Zhang, N. (2019). LncRNA NEF inhibits migration and invasion of HPV-negative cervical squamous cell carcinoma by inhibiting TGF- $\beta$ pathway. Biosci. Rep. 39:BSR20180878. doi: 10.1042/BSR20180878

Kalwa, M., Hänzelmann, S., Otto, S., Kuo, C. C., Franzen, J., Joussen, S., et al. (2016). The lncRNA HOTAIR impacts on mesenchymal stem cells via triple helix formation. Nucleic Acids Res. 44, 10631-10643. doi: 10.1093/nar/gkw802

Kylmaoja, E., Nakamura, M., and Tuukkanen, J. (2016). Osteoclasts and Remodeling Based Bone Formation. Curr. Stem Cell Res. Ther. 11, 626-633. doi: 10.2174/1574888X10666151019115724

Lane, J. M., Russell, L., and Khan, S. N. (2000). Osteoporosis. Clin. Orthop. Relat. Res. 372, 139-150. doi: 10.1097/00003086-200003000-00016

Lei, B., Tian, Z., Fan, W., and Ni, B. (2019). Circular RNA: a novel biomarker and therapeutic target for human cancers. Int. J. Med. Sci. 16, 292-301. doi: 10.7150/ijms.28047

Leighton, R., Watson, J. T., Giannoudis, P., Papakostidis, C., Harrison, A., and Steen, R. G. (2017). Healing of fracture nonunions treated with low-intensity pulsed ultrasound (LIPUS): A systematic review and meta-analysis. Injury 48, 1339-1347. doi: 10.1016/j.injury.2017.05.016

Li, C.-J., Xiao, Y., Yang, M., Su, T., Sun, X., Guo, Q., et al. (2018a). Long noncoding RNA Bmncr regulates mesenchymal stem cell fate during skeletal aging. J. Clin. Investigat. 128, 5251-5266. doi: 10.1172/JCI99044

Li, D., Tian, Y., Yin, C., Huai, Y., Zhao, Y., Su, P., et al. (2019a). Silencing of lncRNA AK045490 Promotes Osteoblast Differentiation and Bone Formation via $\beta$-Catenin/TCF1/Runx2 Signaling Axis. Int. J. Mole. Sci. 20:6229. doi: 10. 3390/ijms20246229

Li, J., and Sun, C. K. (2018). Long noncoding RNA SNHG5 is up-regulated and serves as a potential prognostic biomarker in acute myeloid leukemia. Eur. Rev. Med. Pharmacol. Sci. 22, 3342-3347.

Li, W., Zhong, C., Jiao, J., Li, P., Cui, B., Ji, C., et al. (2017). Characterization of hsa_circ_0004277 as a New Biomarker for Acute Myeloid Leukemia via Circular RNA Profile and Bioinformatics Analysis. Int. J. Mol. Sci. 18:597. doi: 10.3390/ijms18030597

Li, W., Zhu, H. M., Xu, H. D., Zhang, B., and Huang, S. M. (2018b). CRNDE impacts the proliferation of osteoclast by estrogen deficiency in postmenopausal osteoporosis. Eur. Rev. Med. Pharmacol. Sci. 22, 58155821.

Li, X. G., Liu, S. C., Qiao, X. F., Kong, Y., Liu, J. G., Peng, X. M., et al. (2019b). LncRNA MEG3 promotes proliferation and differentiation of osteoblasts through Wnt/ $\beta$-catenin signaling pathway. Eur. Rev. Med. Pharmacol. Sci. 23, 4521-4529.

Li, Y., Zheng, Q., Bao, C., Li, S., Guo, W., Zhao, J., et al. (2015). Circular RNA is enriched and stable in exosomes: a promising biomarker for cancer diagnosis. Cell Res. 25, 981-984. doi: 10.1038/cr.2015.82

Liang, J., Shen, Y. C., Zhang, X. Y., Chen, C., Zhao, H., and Hu, J. (2020). Circular RNA HIPK3 downregulation mediates hydrogen peroxide-induced cytotoxicity in human osteoblasts. Aging 12, 1159-1170. doi: 10.18632/aging.10 2674

Liang, W. C., Ren, J. L., Wong, C. W., Chan, S. O., Waye, M. M., Fu, W. M., et al. (2018). LncRNA-NEF antagonized epithelial to mesenchymal transition and cancer metastasis via cis-regulating FOXA2 and inactivating $\mathrm{Wnt} / \beta$-catenin signaling. Oncogene 37, 1445-1456. doi: 10.1038/s41388-017-0041-y

Liang, W.-C., Fu, W.-M., Wang, Y.-B., Sun, Y.-X., Xu, L.-L., Wong, C.-W., et al. (2016). H19 activates Wnt signaling and promotes osteoblast differentiation by functioning as a competing endogenous RNA. Scientif. Rep. 6, 20121-20121. doi: $10.1038 /$ srep 20121

Ling, L., Hu, H. L., Liu, K. Y., Ram, Y. I., Gao, J. L., and Cao, Y. M. (2019). Long noncoding RNA MIRG induces osteoclastogenesis and bone resorption in osteoporosis through negative regulation of miR-1897. Eur. Rev. Med. Pharmacol. Sci. 23, 10195-10203.

Lisignoli, G., Lambertini, E., Manferdini, C., Gabusi, E., Penolazzi, L., Paolella, F., et al. (2017). Collagen type XV and the 'osteogenic status'. J. Cell Mol. Med. 21, 2236-2244. doi: 10.1111/jcmm.13137

Liu, C., Cao, Z., Bai, Y., Dou, C., Gong, X., Liang, M., et al. (2019a). LncRNA AK077216 promotes RANKL-induced osteoclastogenesis and bone resorption via NFATc1 by inhibition of NIP45. J. Cell. Physiol. 234, 1606-1617. doi: $10.1002 /$ jcp. 27031

Liu, H., Li, D., Zhang, Y., and Li, M. (2018). Inflammation, mesenchymal stem cells and bone regeneration. Histochem. Cell Biol. 149, 393-404. doi: 10.1007/ s00418-018-1643-3

Liu, M., Yang, P., Mao, G., Deng, J., Peng, G., Ning, X., et al. (2019b). Long noncoding RNA MALAT1 as a valuable biomarker for prognosis in osteosarcoma: A systematic review and meta-analysis. Int. J. Surg. 72, 206-213. doi: 10.1016/j. ijsu.2019.11.004

Liu, S. C., Sun, Q. Z., Qiao, X. F., Li, X. G., Yang, J. H., Wang, T. Q., et al. (2019c). LncRNA TUG1 influences osteoblast proliferation and differentiation through the Wnt/ $\beta$-catenin signaling pathway. Eur. Rev. Med. Pharmacol. Sci. 23, 4584-4590.

Liu, X. X., Yang, Y. E., Liu, X., Zhang, M. Y., Li, R., Yin, Y. H., et al. (2019d). A two-circular RNA signature as a noninvasive diagnostic biomarker for lung adenocarcinoma. J. Transl. Med. 17:50. doi: 10.1186/s12967-019-1800-z

Loewen, G., Jayawickramarajah, J., Zhuo, Y., and Shan, B. (2014). Functions of lncRNA HOTAIR in lung cancer. J. Hematol. Oncol. 7:90. doi: 10.1186/s13045014-0090-4

Lucas, T., Bonauer, A., and Dimmeler, S. (2018). RNA Therapeutics in Cardiovascular Disease. Circ. Res. 123, 205-220. doi: 10.1161/CIRCRESAHA. 117.311311

Ma, X., Guo, Z., Gao, W., Wang, J., Liu, Y., Gao, F., et al. (2019). LncRNA-NEF is downregulated in postmenopausal osteoporosis and is related to course of treatment and recurrence. J. Internat. Med. Res. 47, 3299-3306. doi: 10.1177/ 0300060519847854

Manning, J. A., and Kumar, S. (2018). Physiological Functions of Nedd4-2: Lessons from Knockout Mouse Models. Trends Biochem. Sci. 43, 635-647. doi: 10.1016/ j.tibs.2018.06.004

Matsui, M., and Corey, D. R. (2017). Non-coding RNAs as drug targets. Nat. Rev. Drug Discov. 16, 167-179. doi: 10.1038/nrd.2016.117

Mi, B., Xiong, Y., Chen, L., Yan, C., Endo, Y., Liu, Y., et al. (2019). CircRNA AFF4 promotes osteoblast cells proliferation and inhibits apoptosis via the Mir-7223-5p/PIK3R1 axis. Aging 11, 11988-12001. doi: 10.18632/aging.102524

Nie, W. B., Zhao, L. M., Guo, R., Wang, M. X., and Ye, F. G. (2018). Circular RNA circ-NT5C2 acts as a potential novel biomarker for prognosis of osteosarcoma. Eur. Rev. Med. Pharmacol. Sci. 22, 6239-6244.

Oray, M., Abu Samra, K., Ebrahimiadib, N., Meese, H., and Foster, C. S. (2016). Long-term side effects of glucocorticoids. Expert. Opin. Drug Saf. 15, 457-465. doi: $10.1517 / 14740338.2016 .1140743$

Ouyang, Z., Tan, T., Zhang, X., Wan, J., Zhou, Y., Jiang, G., et al. (2019). CircRNA hsa_circ_0074834 promotes the osteogenesis-angiogenesis coupling process in bone mesenchymal stem cells (BMSCs) by acting as a ceRNA for miR-942-5p. Cell Death Dis. 10:932. doi: 10.1038/s41419-019-2161-5

Patil, S., Gao, Y. G., Lin, X., Li, Y., Dang, K., Tian, Y., et al. (2019). The Development of Functional Non-Viral Vectors for Gene Delivery. Int. J. Mol. Sci. 4:20. doi: 10.3390/ijms20215491

Prestwood, K. M., Pilbeam, C. C., and Raisz, L. G. (1995). Treatment of osteoporosis. Annu. Rev. Med. 46, 249-256. doi: 10.1146/annurev.med.46.1.249

Qi, S., Song, Y., Peng, Y., Wang, H., Long, H., Yu, X., et al. (2012). ZEB2 mediates multiple pathways regulating cell proliferation, migration, invasion, and apoptosis in glioma. PLoS One 7, 38842-38842.e. doi: 10.1371/journal.pone. 0038842

Qiao, L., Li, C. G., and Liu, D. (2020).CircRNA_0048211 protects postmenopausal osteoporosis through targeting miRNA-93-5p to regulate BMP2. Eur. Rev. Med. Pharmacol. Sci. 24, 3459-3466. 
Sandhu, G. K., Milevskiy, M. J. G., Wilson, W., Shewan, A. M., and Brown, M. A. (2016). Non-coding RNAs in Mammary Gland Development and Disease. Adv. Exp. Med. Biol. 886, 121-153. doi: 10.1007/978-94-017-7417-8_7

Shang, G., Wang, Y., Xu, Y., Zhang, S., Sun, X., Guan, H., et al. (2018). Long non-coding RNA TCONS_00041960 enhances osteogenesis and inhibits adipogenesis of rat bone marrow mesenchymal stem cell by targeting miR204-5p and miR-125a-3p. J. Cell Physiol. 233, 6041-6051. doi: 10.1002/jcp. 26424

Sözen, T., Özışık, L., and Başaran, N. Ç (2017). An overview and management of osteoporosis. Eur. J. Rheumatol. 4, 46-56. doi: 10.5152/eurjrheum.2016.048

St Laurent, G., Wahlestedt, C., and Kapranov, P. (2015). The Landscape of long noncoding RNA classification. Trends Genet. TIG 31, 239-251. doi: 10.1016/j. tig.2015.03.007

Sun, X., Yuan, Y., Xiao, Y., Lu, Q., Yang, L., Chen, C., et al. (2018). Long noncoding RNA, Bmcob, regulates osteoblastic differentiation of bone marrow mesenchymal stem cells. Biochem. Biophys. Res. Commun. 506, 536-542. doi: 10.1016/j.bbrc.2018.09.142

Tabatabaei-Malazy, O., Salari, P., Khashayar, P., and Larijani, B. (2017). New horizons in treatment of osteoporosis. Daru 25:2. doi: 10.1186/s40199-0170167-z

Wan, G., Liu, Y., Han, C., Zhang, X., and Lu, X. (2014). Noncoding RNAs in DNA repair and genome integrity. Anti. Redox. Sign. 20, 655-677. doi: 10.1089/ars. 2013.5514

Wang, C.-G., Liao, Z., Xiao, H., Liu, H., Hu, Y.-H., Liao, Q.-D., et al. (2019a). LncRNA KCNQ1OT1 promoted BMP2 expression to regulate osteogenic differentiation by sponging miRNA-214. Exper. Mole. Pathol. 107, 77-84. doi: 10.1016/j.yexmp.2019.01.012

Wang, H., Li, Y. K., Cui, M., Liu, L. H., Zhao, L. M., and Wang, X. M. (2020a). Effect of lncRNA AK125437 on postmenopausal osteoporosis rats via MAPK pathway. Eur. Rev. Med. Pharmacol. Sci. 24, 2173-2180.

Wang, H., Zhao, W., Tian, Q. J., Xin, L., Cui, M., and Li, Y. K. (2020b). Effect of lncRNA AK023948 on rats with postmenopausal osteoporosis via PI3K/AKT signaling pathway. Eur. Rev. Med. Pharmacol. Sci. 24, 2181-2188.

Wang, Q., Li, Y., Zhang, Y., Ma, L., Lin, L., Meng, J., et al. (2017a). LncRNA MEG3 inhibited osteogenic differentiation of bone marrow mesenchymal stem cells from postmenopausal osteoporosis by targeting miR-133a3p. Biomed. Pharmacother. 89, 1178-1186. doi: 10.1016/j.biopha.2017. 02.090

Wang, X. B., Li, P. B., Guo, S. F., Yang, Q. S., Chen, Z. X., Wang, D., et al. (2019b). circRNA_0006393 promotes osteogenesis in glucocorticoid-induced osteoporosis by sponging miR-145-5p and upregulating FOXO1. Mol. Med. Rep. 20, 2851-2858. doi: 10.3892/mmr.2019.10497

Wang, Y., Liu, W., Liu, Y., Cui, J., Zhao, Z., Cao, H., et al. (2018a). Long noncoding RNA H19 mediates LCoR to impact the osteogenic and adipogenic differentiation of mBMSCs in mice through sponging miR-188. J. Cell. Physiol. 233, 7435-7446. doi: 10.1002/jcp.26589

Wang, Y., Luo, T.-B., Liu, L., and Cui, Z.-Q. (2018b). LncRNA LINC00311 Promotes the Proliferation and Differentiation of Osteoclasts in Osteoporotic Rats Through the Notch Signaling Pathway by Targeting DLL3. Cell. Physiol. Biochem. 47, 2291-2306. doi: 10.1159/000491539

Wang, Y., Wang, K., Zhang, L., Tan, Y., Hu, Z., Dang, L., et al. (2020c). Targeted overexpression of the long noncoding RNA ODSM can regulate osteoblast function in vitro and in vivo. Cell Death Dis. 11:133. doi: 10.1038/s41419-0202325-3

Wang, Y., Wu, C., Zhang, Y., Yang, Y., Ren, Z., Lammi, M. J., et al. (2020d). Screening for differentially expressed circRNA between Kashin-Beck disease and osteoarthritis patients based on circRNA chips. Clin. Chim. Acta 501, 92-101. doi: 10.1016/j.cca.2019.10.026

Wang, Y., Zhou, Q., and Ma, J. J. (2018c). High expression of lnc-CRNDE presents as a biomarker for acute myeloid leukemia and promotes the malignant progression in acute myeloid leukemia cell line U937. Eur. Rev. Med. Pharmacol. Sci. 22, 763-770.

Wang, Z., Tan, M., Chen, G., Li, Z., and Lu, X. (2017b). LncRNA SOX2-OT is a novel prognostic biomarker for osteosarcoma patients and regulates osteosarcoma cells proliferation and motility through modulating SOX2. IUBMB Life 69, 867-876. doi: 10.1002/iub.1681

Weinstein, R. S. (2012). Glucocorticoid-induced osteoporosis and osteonecrosis. Endocrinol. Metab. Clin. North Am. 41, 595-611. doi: 10.1016/j.ecl.2012.04.004
Wen, J., Guan, Z., Yu, B., Guo, J., Shi, Y., and Hu, L. (2020). Circular RNA hsa_circ_0076906 competes with OGN for miR-1305 biding site to alleviate the progression of osteoporosis. Int. J. Biochem. Cell Biol. 122:105719. doi: 10.1016/j.biocel.2020.105719

Whittier, X., and Saag, K. G. (2016). Glucocorticoid-induced Osteoporosis. Rheum. Dis. Clin. North Am. 42, 177-189. doi: 10.1016/j.rdc.2015.08.005

Williams, B. O. (2017). LRP5: From bedside to bench to bone. Bone 102, 26-30. doi: 10.1016/j.bone.2017.03.044

Wu, J., Ren, W., Zheng, Z., Huang, Z., Liang, T., Li, F., et al. (2020). Mmu_circ_003795 regulates osteoblast differentiation and mineralization in MC3T3-E1 and MDPC23 by targeting COL15A1. Mol. Med. Rep. 22, $1737-$ 1746. doi: $10.3892 / \mathrm{mmr} .2020 .11264$

Wu, J., Zhao, J., Sun, L., Pan, Y., Wang, H., and Zhang, W.-B. (2018a). Long non-coding RNA H19 mediates mechanical tension-induced osteogenesis of bone marrow mesenchymal stem cells via FAK by sponging miR-138. Bone 108, 62-70. doi: 10.1016/j.bone.2017.12.013

Wu, Q.-Y., Li, X., Miao, Z.-N., Ye, J.-X., Wang, B., Zhang, F., et al. (2018b). Long Non-coding RNAs: A New Regulatory Code for Osteoporosis. Front. Endocrinol. 9, 587-587. doi: 10.3389/fendo.2018.00587

Wu, Y., Jiang, Y., Liu, Q., and Liu, C. Z. (2019). IncRNA H19 promotes matrix mineralization through up-regulating IGF1 by sponging miR-185-5p in osteoblasts. BMC Mol. Cell Biol. 20:48. doi: 10.1186/s12860-019-0230-3

Wu, Z. H., Huang, K. H., Liu, K., Wang, G. T., and Sun, Q. (2018c). DGCR5 induces osteogenic differentiation by up-regulating Runx2 through miR-30d5p. Biochem. Biophys. Res. Commun. 505, 426-431. doi: 10.1016/j.bbrc.2018.09. 033

Xi, J. C., Zang, H. Y., Guo, L. X., Xue, H. B., Liu, X. D., Bai, Y. B., et al. (2015). The PI3K/AKT cell signaling pathway is involved in regulation of osteoporosis. J. Recept Sign. Transduct. Res. 35, 640-645. doi: 10.3109/10799893.2015. 1041647

Yang, L., Li, Y., Gong, R., Gao, M., Feng, C., Liu, T., et al. (2019). The Long Noncoding RNA-ORLNC1 Regulates Bone Mass by Directing Mesenchymal Stem Cell Fate. Mole. Ther. J. Am. Soc. Gene Ther. 27, 394-410. doi: 10.1016/j.ymthe. 2018.11.019

Ye, Y., Shen, A., and Liu, A. (2019). Long non-coding RNA H19 and cancer: A competing endogenous RNA. Bull. Cancer 106, 1152-1159. doi: 10.1016/j. bulcan.2019.08.011

Yi, J., Liu, D., and Xiao, J. (2019). LncRNA MALAT1 sponges miR-30 to promote osteoblast differentiation of adipose-derived mesenchymal stem cells by promotion of Runx2 expression. Cell Tissue Res. 376, 113-121. doi: 10.1007/ s00441-018-2963-2

Yin, C., Tian, Y., Yu, Y., Wang, H., Wu, Z., Huang, Z., et al. (2019). A novel long noncoding RNA AK016739 inhibits osteoblast differentiation and bone formation. J. Cell. Physiol. 234, 11524-11536. doi: 10.1002/jcp.27815

Yu, H., Zhou, W., Yan, W., Xu, Z., Xie, Y., and Zhang, P. (2019). LncRNA CASC11 is upregulated in postmenopausal osteoporosis and is correlated with TNF- $\alpha$. Clin. Intervent. Aging 14, 1663-1669. doi: 10.2147/CIA.S205796

Yu, L., and Liu, Y. (2019). circRNA_0016624 could sponge miR-98 to regulate BMP2 expression in postmenopausal osteoporosis. Biochem. Biophys. Res. Commun. 516, 546-550. doi: 10.1016/j.bbrc.2019.06.087

Yu, L., Xia, K., Cen, X., Huang, X., Sun, W., Zhao, Z., et al. (2020). DNA methylation of noncoding RNAs: new insights into osteogenesis and common bone diseases. Stem Cell Res. Ther. 11:109. doi: 10.1186/s13287-02001625-7

Zhai, N., Lu, Y., Wang, Y., Ren, X., and Han, J. (2018). Circular RNAs and hereditary bone diseases. Intractab. Rare Dis. Res. 7, 1-6. doi: 10.5582/irdr.2018. 01013

Zhang, C., Zhu, Y., Liu, Y., Zhang, X., Yue, Q., Li, L., et al. (2019a). SEMA3BAS1-inhibited osteogenic differentiation of human mesenchymal stem cells revealed by quantitative proteomics analysis. J. Cell Physiol. 234, 2491-2499. doi: $10.1002 /$ jcp. 26776

Zhang, H. L., Du, X. Y., and Dong, Q. R. (2019b). LncRNA XIXT promotes osteogenic differentiation of bone mesenchymal stem cells and alleviates osteoporosis progression by targeting miRNA-30a-5p. Eur. Rev. Med. Pharmacol. Sci. 23, 8721-8729.

Zhang, M., and Du, X. (2016). Noncoding RNAs in gastric cancer: Research progress and prospects. World J. Gastroenterol. 22, 6610-6618. doi: 10.3748/ wjg.v22.i29.6610 
Zhang, N., Hu, X., He, S., Ding, W., Wang, F., Zhao, Y., et al. (2019c). LncRNA MSC-AS1 promotes osteogenic differentiation and alleviates osteoporosis through sponging microRNA-140-5p to upregulate BMP2. Biochem. Biophys. Res. Commun. 519, 790-796. doi: 10.1016/j.bbrc.2019. 09.058

Zhang, R. F., Liu, J. W., Yu, S. P., Sun, D., Wang, X. H., Fu, J. S., et al. (2019d). LncRNA UCA1 affects osteoblast proliferation and differentiation by regulating BMP-2 expression. Eur. Rev. Med. Pharmacol. Sci. 23, 6774-6782.

Zhang, X. Y., Shan, H. J., Zhang, P., She, C., and Zhou, X. Z. (2018). LncRNA EPIC1 protects human osteoblasts from dexamethasone-induced cell death. Biochem. Biophys. Res. Commun. 503, 2255-2262. doi: 10.1016/j.bbrc.2018. 06.146

Zhang, X., Hamblin, M. H., and Yin, K. J. (2017). The long noncoding RNA Malat1: Its physiological and pathophysiological functions. RNA Biol. 14, 1705-1714. doi: 10.1080/15476286.2017.1358347

Zhang, Y., Chen, B., Li, D., Zhou, X., and Chen, Z. (2019e). LncRNA NEAT1/miR-29b-3p/BMP1 axis promotes osteogenic differentiation in human bone marrow-derived mesenchymal stem cells. Pathol. Res. Pract. 215, 525-531. doi: $10.1016 /$ j.prp.2018.12.034

Zhang, Z., Zhang, J., and Xiao, J. (2014). Selenoproteins and selenium status in bone physiology and pathology. Biochim. Biophys. Acta 1840, 3246-3256. doi: 10.1016/j.bbagen.2014.08.001

Zheng, J., Huang, Y., Li, Y., Lai, J., Chen, C., Yi, C., et al. (2020). IncRNA-TINCR Functions as a Competitive Endogenous RNA to Regulate the Migration of Mesenchymal Stem Cells by Sponging miR-761. Biomed. Res. Int. 2020:9578730. doi: $10.1155 / 2020 / 9578730$
Zheng, S., Wang, Y. B., Yang, Y. L., Chen, B. P., Wang, C. X., Li, R. H., et al. (2019). LncRNA MALAT1 inhibits osteogenic differentiation of mesenchymal stem cells in osteoporosis rats through MAPK signaling pathway. Eur. Rev. Med. Pharmacol. Sci. 23, 4609-4617.

Zhu, X. X., Yan, Y. W., Chen, D., Ai, C. Z., Lu, X., Xu, S. S., et al. (2016). Long noncoding RNA HoxA-AS3 interacts with EZH2 to regulate lineage commitment of mesenchymal stem cells. Oncotarget 7, 63561-63570. doi: 10.18632/oncotarget. 11538

Zhuang, W., Ge, X., Yang, S., Huang, M., Zhuang, W., Chen, P., et al. (2015). Upregulation of IncRNA MEG3 Promotes Osteogenic Differentiation of Mesenchymal Stem Cells From Multiple Myeloma Patients By Targeting BMP4 Transcription. Stem Cells 33, 1985-1997. doi: 10.1002/stem.1989

Zvereva, M. I., Shcherbakova, D. M., and Dontsova, O. A. (2010). Telomerase: structure, functions, and activity regulation. Biochemistry 75, 1563-1583. doi: $10.1134 /$ S0006297910130055

Conflict of Interest: The authors declare that the research was conducted in the absence of any commercial or financial relationships that could be construed as a potential conflict of interest.

Copyright (C) 2020 Patil, Dang, Zhao, Gao and Qian. This is an open-access article distributed under the terms of the Creative Commons Attribution License (CC BY). The use, distribution or reproduction in other forums is permitted, provided the original author(s) and the copyright owner(s) are credited and that the original publication in this journal is cited, in accordance with accepted academic practice. No use, distribution or reproduction is permitted which does not comply with these terms. 